\title{
The velocity of spatial population expansion
}

\author{
F. van den Bosch ${ }^{1}$, J. A. J. Metz ${ }^{1}$, and O. Diekmann ${ }^{1,2}$ \\ ' Institute of Theoretical Biology, State University of Leiden, Kaiserstraat 63, \\ 2311 GP Leiden, The Netherlands \\ ${ }^{2}$ Centre for Mathematics and Computer Science, Kruislaan 413, 1098 SJ Amsterdam, \\ The Netherlands
}

\begin{abstract}
We consider the velocity with which an invading population spreads over space. For a general linear model, originally due to Diekmann and Thieme, it is shown that the asymptotic velocity of population expansion can be calculated if information is available on: (i) the net-reproduction, $R_{0}$; i.e. the expected number of offspring produced by one individual throughout its life, and (ii) the (normalized) reproduction-and-dispersal kernel, $\beta(a, x-\xi)$; i.e. the density of newborns produced per unit of time at position $x$ by an individual of age $a$ born at $\xi$. By means of numerical examples we study the effect of the net-reproduction and the shape of the reproductionand-dispersal kernel on the velocity of population expansion. The reproduction-and-dispersal kernel is difficult to measure in full. This leads us to derive approximation formulas in terms of easily measurable parameters. The relation between the velocity of population expansion calculated from the general model and that from the Fisher/Skellam diffusion model is discussed. As a final step we use the model to analyse some real-life examples, thus showing how it can be put to work.
\end{abstract}

Key words: Space time - Integral equation - Dispersal - Asymptotic velocity of propagation - Approximation formulae - Cumulant generating function - Fisher/Skellam diffusion model.

\section{Introduction}

Once upon a time a rich Czech prince went muskrat hunting in Alaska. He liked this so much that he took five muskrats back home and released them at his country-seat near Prague. These introduced individuals and their offspring started to spread, and today muskrat populations are established throughout Europe. 
This is one of many well-documented examples of biological invasions. A biological invasion can loosely be defined as a (on an evolutionary time scale) sudden extension of a populations range. Often such invasions are induced by man. Sometimes they are the result of natural extensions of range. An epidemic of an infectious disease can be viewed as the expansion of a population of disease organisms and therefore also falls in the category of invasions.

Invading species often have an influence on the ecosystem or the agricultural system. From the point of view of the economist or the nature conservationist, this influence is often considered to be negative. This fact led the Scientific Committee on Problems of the Environment (SCOPE) to organize a program on 'The Ecology of Biological Invasions' (Levin, in press; Mooney and Drake 1986; Kornberg and Williamson 1987; Anonymous 1985). This programme addresses several questions concerning invasions, ranging from the invadability of ecosystems to the development of management systems to prevent unwanted invasions.

An invasion which starts at a certain place often does not have an immediate effect at another, distant, place. The spatial component is, therefore, frequently of considerable importance. After the pioneering work of Fisher (1937), Skellam (1951), Kendall (1965) and Mollison $(1972,1977)$, Diekmann $(1978,1979)$ and Thieme (1977a,b; 1979a,b) developed and studied a rather general model for the spatial spread of populations. The velocity with which an invading population spreads over space obviously depends on the population dynamical attributes of the individuals making up that population. Using the Diekmann/Thieme approach we show in this paper how individual behaviour and the velocity of population expansion are related.

Although the models we present are of a general nature, we will use a terminology proper to animal species in the main text. In some examples we use a different, but compatible, terminology proper to the species under consideration. For species with two sexes we consider females only.

This paper bridges part of the gap between, on the one hand, some abstract theorems about the velocity of population expansion in the mathematical and biomathematical literature and, on the other hand, data concerning real biological invasions. The paper is written for mathematically inclined biologists and mathematicians working on practical biological problems. Heuristic arguments and formal calculations abound in the paper. No proofs are given.

\section{Diffusion models}

To develop some intuition, terminology and notation we start with a discussion about diffusion models. The simplest diffusion equation, modelling spatial spread in two dimensions reads

$$
\frac{\partial n}{\partial t}=\frac{1}{2} s\left(\frac{\partial^{2} n}{\partial x_{1}^{2}}+\frac{\partial^{2} n}{\partial x_{2}^{2}}\right)+f(n) n
$$

where $n(t, x)$ is the population density at position $x=\left(x_{1}, x_{2}\right)$ at time $t, s$ is the 'diffusion constant' indicating the rate of random movement and $f(n)$ is the per 
capita 'population growth rate' as a function of local population density. We assume that $f^{\prime}(n) \leqslant 0$ for all $n \geqslant 0$.

First consider the linear equation obtained by putting $f(n)=f(0)$, for all $n$. The solution, with initial condition $n(0, x)=\delta(x)$, is given explicitly by

$$
n(t, x)=\frac{1}{2 \pi s t} \exp \left(-\frac{|x|^{2}}{2 s t}+f(0) t\right) .
$$

It is easily seen that for any $\varepsilon(>0)$

$$
n(t, x) \underset{t \rightarrow \infty}{\longrightarrow} \begin{cases}0 & \text { if }|x|>\left(C_{0}+\varepsilon\right) t \\ \infty & \text { if }|x|<\left(C_{0}-\varepsilon\right) t\end{cases}
$$

where

$$
C_{0}=\sqrt{2 s f(0)}
$$

(2.3) states that if one travels in a straight line away from the origin with a velocity larger (smaller) than $C_{0}$, one will, in the long run, observe a population with density zero (infinity). In this sense $C_{0}$ is the asymptotic velocity of population expansion. Note that, due to the rotational symmetry, the value of $C_{0}$ does not depend on the direction. From (2.2) it is also seen that contours of equal population density behave like expanding circles. Contours of equal rate of population change, $\partial n / \partial t$, behave in the same way.

The non-linear model (2.1) cannot be solved explicitly. Now consider socalled travelling plane wave solutions, i.e. solutions of the form

$$
n(t, x)=\tilde{n}(x \cdot v-C t)
$$

where $C(\geqslant 0)$ is the velocity of the wave and $v$ is a unit vector giving the direction of movement. Such a solution can be visualized as a function of $x$, which has, at a given time, a constant value on lines $x \cdot v=$ constant. This function of $x$ is shifted with constant velocity, $C$, in the direction $v$ without any change in shape. It can be shown (e.g. Fisher 1937; Aronson and Weinberger 1975; Hadeler and Rothe 1975; Diekmann and Temme 1976) that (2.1) has travelling plane wave solutions for every $C \geqslant C_{0}$, where $C_{0}$ is defined by (2.4). The velocity $C_{0}$ is called the minimal wave velocity.

Aronson and Weinberger $(1975,1978)$ show that, at least for initial conditions with a bounded spatial support, $C_{0}$ is still the asymptotic velocity of population expansion in the sense of (2.3). The density dependence has not changed it! (In this situation we, of course, have to replace the ' $\infty$ ' at the right-hand side of $(2.3)$ by $\hat{n}$, where $\hat{n}$ is the solution of $f(\hat{n})=0$.) (Various more subtle aspects of the convergence of solutions of (2.1) to travelling wave solutions are studied by, e.g., Kolmogorov et al. (1937), and Bramson (1983).) Note that founder populations always have a bounded spatial support.

A physical analog makes the fact that the minimal wave velocity is also the asymptotic velocity intuitively clear. Imagine a large number of firepots aligned such that they can kindle each other. Attach to each pot a piece of slowmatch that can set fire to that particular pot. We can now create (the illusion of) a travelling wave by setting fire to all pieces of slowmatch simultaneously but at 
distances from the firepot which increase linearly with the serial number of the pot. The steepness of this linear relation determines the wave velocity. However, if we try to make the velocity too small cross kindling takes over. Consequently the velocity produced by cross kindling necessarily corresponds to the lowest possible wave velocity. If we set fire to only a small number of slowmatches at one end of the line of firepots (the analogue of a founder population with bounded support), this will always produce a wave of burning firepots with the minimal velocity.

Sometimes the dispersion of individuals is not isotropic (rotationally symmetric). Diffusion rates may be different in the various directions or there may be a systematic movement of individuals in a certain direction. In the rotationally symmetric (linear) case the circularity of contours of equal population density makes that the (minimal) velocity of a travelling plane wave solution is equal to the asymptotic velocity of population expansion along any straight line from the origin. In the non-rotationally symmetric case contours of equal population density are not circular, both the minimal plane wave velocity and the asymptotic velocity depend on the direction, and they are not necessarily equal (Fig. 1). As a simple example we consider the linear diffusion model:

$$
\frac{\partial n}{\partial t}=-\sum_{i} m_{i} \frac{\partial n}{\partial x_{i}}+\frac{1}{2} \sum_{i j} s_{i j} \frac{\partial^{2} n}{\partial x_{i} \partial x_{j}}+f(0) n ; \quad i, j=1,2 \quad \text { and } \quad s_{12}=s_{21}
$$

With initial condition $n\left(0, x_{1}, x_{2}\right)=\delta(x)$, the solution of $(2.6)$ is

$$
n\left(t, x_{1}, x_{2}\right)=\frac{\exp \left[f(0) t-\frac{1}{2 t}(X-M t)^{T} S^{-1}(X-M t)\right]}{2 \pi t \sqrt{\operatorname{det} S}}
$$

where $X=\left(x_{1}, x_{2}\right)^{T}, M=\left(m_{1}, m_{2}\right)^{T}, S=\left(s_{i j}\right)$ and $\operatorname{det} S$ is the determinant of the matrix $S$. Contours of equal population density are for $t \rightarrow \infty$ given by

$$
(X-M t)^{T} S^{-1}(X-M t)=2 f(0) t^{2} .
$$

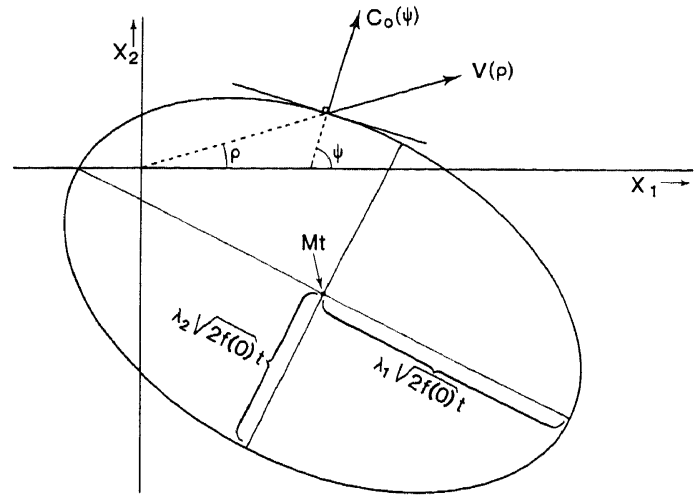

Fig. 1. Contours of equal population density for a (linear) diffusion model with convection (Eq. (2.6)). The contours are expanding ellipses. The figure also illustrates the difference between the velocity of a plane population front, $C_{0}(\psi)$, and the velocity of population expansion along a straight line from the origin, $V(\varrho)$ 
These are expanding ellipses with their centre at $M t$, and axes in the direction of the eigenvectors of $S$. The length of the axes is given by $\lambda_{i} \sqrt{2 f(0)} t$, where the $\lambda_{i}$ are the corresponding eigenvalues (Fig. 1). From this, the asymptotic velocity, $V(\varrho)$, of population expansion along a straight line from the origin making an angle $\varrho$ with the $x_{1}$-axis is

$$
V(\varrho)=\frac{-\left(s_{12} m_{2}-s_{22} m_{1}\right) \cos \varrho+\left(s_{12} m_{1}-s_{11} m_{2}\right) \sin \varrho \pm \sqrt{Q(\varrho)}}{s_{22} \cos ^{2} \varrho-2 s_{12} \cos \varrho \sin \varrho+s_{11} \sin ^{2} \varrho}
$$

where

$$
\begin{aligned}
Q(\varrho)= & {\left[\left(s_{12} m_{2}-s_{22} m_{1}\right) \cos \varrho+\left(s_{12} m_{1}-s_{11} m_{2}\right) \sin \varrho\right]^{2} } \\
& -\left(s_{22} \cos ^{2} \varrho-2 s_{12} \cos \varrho \sin \varrho+s_{11} \sin ^{2} \varrho\right) \\
& \cdot\left[s_{22} m_{1}^{2}-2 s_{12} m_{1} m_{2}+s_{11} m_{2}^{2}+2\left(s_{12}^{2}-s_{11} s_{22}\right) f(0)\right]
\end{aligned}
$$

The collection of vectors $V(\varrho)$ gives the shape of the contour of equal population density and is presented as the ellipse in Fig. 1 with $t=1$.

On the other hand, the asymptotic velocity of a plane wave front moving in the direction $x_{1}=(y \cos \psi), x_{2}=(y \sin \psi)$ can be calculated from the following one-dimensional version of (2.6).

$$
\frac{\partial n}{\partial t}=-m \frac{\partial n}{\partial y}+\frac{1}{2} s \frac{\partial^{2} n}{\partial y^{2}}+f(0) n
$$

with coefficients

$$
m=(\cos \psi, \sin \psi) M ; \quad s=(\cos \psi, \sin \psi) S\left(\begin{array}{c}
\cos \psi \\
\sin \psi
\end{array}\right) .
$$

The solution of (2.10) corresponding to a Dirac mass initial condition is given by (2.2) if we substitute $|y-m t|$ for $|x|$. This gives

$$
C_{0}(\psi)=\left(m_{1} \cos \psi+m_{2} \sin \psi\right) \pm \sqrt{2 f(0) \tilde{P}(\psi)},
$$

where

$$
\tilde{P}(\psi)=s_{11} \cos ^{2} \psi+2 s_{12} \cos \psi \sin \psi+s_{22} \sin ^{2} \psi
$$

The solution of (2.10) can be visualized as a (moving) function of $y$ which has the shape of a Gaussian density, the area of which increases exponentially and which shifts with velocity $m$ in the positive direction (Fig. 2). It is obvious that when $m$ is large enough, compared to $s$ and $f(0)$, the population does not build up in the negative direction. The asymptotic velocity in that direction is then negative. For instance, consider Eq. (2.12); when $m_{1}>\sqrt{2 f(0) \tilde{P}(\psi)}$, there is a (large) positive $C_{0}(\psi)$ if one looks in the $\psi=0$ direction and a (small) negative $C_{0}(\psi)$ if one looks in the opposite direction $(\psi=\pi)$. This is illustrated in Fig. $2 \mathrm{~b}$. Similarly, when $M$ is large enough, the ellipse (2.8) does not enclose the origin.

Diffusion equations, such as (2.1), are often applied in studies on the spatial spread of populations (Okubo 1986; Williamson and Brown 1986; Lubina and Levin 1988; Andow et al., preprint; Källén et al. 1985; Caughley 1970; Watt 1968; Noble 1974). Although much insight is gained, there is an inherent 


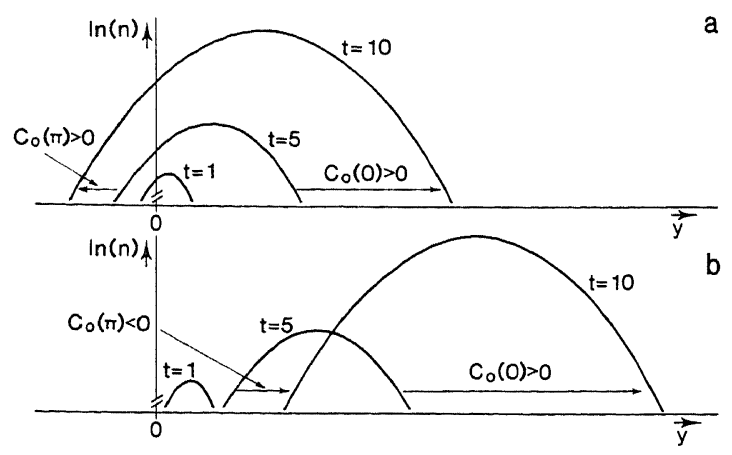

a

Fig. 2a,b. The solution of a linear diffusion model with convection (Eq. (2.10)). Horizontal: distance $y$; vertical: number of individuals, $n$ (log scale). In a the convection velocity is small compared to the diffusion coeffirient and the population growth rate. The velocities of population expansion in both the positive and the negative $y$ direction are therefore positive; in b convection is much larger, resulting in a negative velocity in the negative $y$ direction

draw-back to the use of the diffusion equation formulation. These equations make very specific assumptions about the processes at the individual level, to wit: (i) every individual moves at random throughout its life, (ii) the reproductionand death-rate of the individuals only depend on their local environment (be it constant, $f(0)$, or dependent on population density, $f(n)$ ). The life history of species generally is more complicated. For example, reproduction- and deathrates may depend on age, or an individual may settle down permanently on a breeding ground at the end of its juvenile period. It is interesting to know how the velocity of population expansion is related to the life history of the individuals comprising the population.

In practical applications, quantities like the probability to survive to a certain age and the settlement pattern of juveniles can be obtained experimentally. The question then is how we can calculate the velocity of population expansion from such experimentally observed quantities.

In the following section we shall present a modelling framework that allows us to answer this question.

\section{The linear model}

\subsection{The integral equation for the birth rate}

We assume a constant environment. This entails a fixed relation between age and the average population dynamical behaviour (rate of giving birth, probability of dying, etc.) of an individual (provided 'individuals are born equal'). Therefore we can write down an age structured model (compare Metz and Diekmann 1986, chap. IV).

Individual behaviour. A 'general' model should incorporate as few assumptions as possible about reproduction and dispersal at the individual level. We define the function $\tilde{B}(a, x, \xi)$ to be the density of newborns produced per unit of time at position $x$ by an individual of age $a$ born at $\xi$. $\tilde{B}$ will be called the reproductionand-dispersal kernel. 
Throughout this paper we assume the habitat to be homogeneous, i.e.

$$
\widetilde{B}(a, x, \xi)=B(a, x-\xi) \text {. }
$$

When $B$ is rotationally symmetric it is a function of the distance $|x-\xi|$ only.

The function $B$ can be normalized by defining

$$
\beta(a, x-\xi)=R_{0}^{-1} B(a, x-\xi),
$$

where

$$
R_{0}=\int_{0}^{\infty} \int_{\mathbb{R}^{2}} B(a, x) d x d a
$$

is the expected total number of offspring produced by one individual throughout its whole life. This quantity is known as the net-reproduction. We assume that $B$ is such that this integral exists and is finite. It is obvious that a necessary and sufficient condition for a population to grow if it is infinitesimally small is $R_{0}>1$. Throughout this paper we restrict our attention to this situation. Note that $\beta$ can be interpreted as a probability density. $\beta$ will be called the normalized reproduction-and-dispersal kernel.

The marginal density,

$$
\beta^{0}(a)=\int_{\mathbb{R}^{2}} \beta(a, x) d x,
$$

is the probability density of a random variable called 'the age-at-child-bearing'. A biological interpretation of $\beta^{0}$ can be found in Metz and Diekmann (1986, p. 153). $\beta^{0}(a)$ will be called the reproduction kernel. The marginal density,

$$
D(x)=\int_{0}^{\infty} \beta(a, x) d a,
$$

is called the dispersal density. It is the probability density of the place of birth of an average offspring from an individual which was born itself at the origin.

We shall frequently assume that reproduction and dispersal are statistically independent, i.e.:

$$
\beta(a, x)=\beta^{0}(a) \cdot D(x) .
$$

Note the difference between statistical independence and the mechanistic independence incorporated in, for example, the diffusion models. Although dispersal rates in such models are independent of age the resulting accrued dispersal is a function of age and (3.4) does not apply.

Example 1. For mammals and birds $\tilde{B}$ incorporates both the demographic and the dispersal characteristics of an individual. The demographic characteristics are the two basic life-table statistics: (i) the probability that an individual is still alive at age $a, L(a)$, usually called the age specific suvivorship and (ii) the rate of offspring production of an individual at age $a, m(a)$. The dispersal characteristic is the conditional dispersal density, $D^{*}(a, x, \xi \mid$ alive $)$, defined as the probability that an individual born at $\xi$ is living at $x$ at age $a$, given that it is still alive. The 
reproduction-and-dispersal kernel is given by

$$
\widetilde{B}(a, x, \xi)=L(a) m(a) D^{*}(a, x, \xi \mid \text { alive }) .
$$

When individuals of a species first disperse and then settle down permanently on their breeding-ground at the end of the juvenile period, (3.4) applies.

Example 2. For infectious diseases the definition of 'individual' requires some care. For some diseases one pathogen individual is equivalent to one individual in our model. In other cases a complete population of pathogen individuals localized in one host is equivalent to one 'individual' in our model. For instance, one fox is one 'individual' if we consider rabies, even though a rabid fox contains millions of rabies viruses. For any particular disease, 'individual' has to be chosen such that we can assume that, once infected, the course of the individual's infectivity is an autonomous process. Define the infectivity $I(a)$, to be the number of new infections caused per unit of time by an individual which has been infected time $a$ ago. $I(a)$ includes the probability that the individual is still alive.

The precise biological interpretation of the dispersal density, $D^{*}(a, x, \xi \mid$ alive $)$ depends on the transfer mechanism of the pathogen. When disease is transmitted through physical contacts, $D^{*}$ is the probability that a host having its centre of activity at $\xi$ infects, at age of illness $a$, a host having its centre of activity at $x$. When the infection is transmitted through air-borne spores, $D$ is the probability that a spore released at $\xi$, infects a suscept at $x$.

By definition, $\tilde{B}(a, x, \xi)=I(a) D^{*}(a, x, \xi \mid$ alive $)$.

When the spatial behaviour of an individual does not change during the course of its illness (3.4) applies. When the dispersal of fungal spores takes place at a much shorter time scale than the time scale of spore production we can again use (3.4).

The birth rate equation. Denote by $b(t, x)$ the number of births per unit of area and per unit of time at position $x$ at time $t$. This quantity equals the sum of all current births at $x$ from parents of all possible ages born at all possible places. The current births due to parents of age $a$ born at $\xi$ is equal to the number (actually the density) of parents born time $a$ ago at $\xi, b(t-a, \xi)$, times their per capita rate of offspring production at $x, R_{0} \beta(a, x-\xi)$. The population equation therefore takes the form:

$$
b(t, x)=R_{0} \int_{0}^{\infty} \int_{\mathbb{R}^{2}} b(t-a, \xi) \beta(a, x-\xi) d \xi d a .
$$

Note that this equation is a spatial variant of the renewal equation, originally due to Lotka, which is used in demography.

Remark 1. Actually the time integral extends backwards only till the time the population was founded and we also have to describe the influence of the founder population. However, in this paper we are interested in the asymptotic behaviour of the model and we can therefore restrict our attention to (3.5). 
Remark 2. The total number of individuals at position $x$ at time $t, n(t, x)$, can be calculated from

$$
n(t, x)=\int_{0}^{\infty} \int_{\mathbb{R}^{2}} b(t-a, \xi) \mathscr{L}(a, x-\xi) d \xi d a,
$$

where $\mathscr{L}(a, x-\xi)$ is the probability that an individual of age $a$ born at $\xi$ is alive and living at $x$. Since no individual lives forever it is reasonable to assume that $\mathscr{L}$ is such that this integral exists and is finite.

\subsection{The velocity of population expansion}

Plane wave solutions. Travelling plane wave solutions of (3.5) have the form

$$
b(t, x)=\tilde{b}(C t-x \cdot v)
$$

where $v=(\cos \psi, \sin \psi)^{T}$ for some $\psi \in[0,2 \pi)$. Substitution of the trial solution,

$$
\tilde{b}(C t-x \cdot v)=\zeta \exp (\lambda(C t-x \cdot v))
$$

leads to the characteristic equation

$$
L(C, \lambda)=1
$$

where

$$
L(C, \lambda)=R_{0} \int_{0}^{\infty} \int_{\mathbb{R}^{2}} e^{-\lambda(C a-\xi \cdot v)} \beta(a, \xi) d \xi d a .
$$

Applying the transformation $\quad\left(\xi_{1}^{\prime}=\xi_{1} \cos \psi+\xi_{2} \sin \psi ; \xi_{2}^{\prime}=-\xi_{1} \sin \psi+\right.$ $\left.\xi_{2} \cos \psi\right)$ and immediately dropping the primes yields

$$
\left.L(C, \lambda)=R_{0} \int_{0}^{\infty} \int_{-\infty}^{\infty} e^{-\lambda\left(C a-\xi_{1}\right)} \tilde{\beta(} a, \xi_{1}\right) d \xi_{1} d a,
$$

where

$$
\tilde{\beta}\left(a, \xi_{1}\right)=\int_{-\infty}^{\infty} \beta\left(a ; \xi_{1} \cos \psi-\xi_{2} \sin \psi, \xi_{1} \sin \psi+\xi_{2} \cos \psi\right) d \xi_{2} .
$$

Note that $\tilde{\beta}$ is the marginal-distribution of $\beta$ over the line $x \cdot v=0$. When $\lambda$ and $C$ are chosen such that (3.8) holds, there is a travelling wave solution of exponential form, (3.7). We can restrict our attention to $\lambda \geqslant 0$ only, as any relations for $\lambda<0$ are automatically found when we consider (3.8) with $v$ replaced by $-v$ and $C$ by $-C$. The interpretation is given by the following observation: Consider someone looking in a certain direction and observing a travelling wave characterized by $(\lambda, C)$. Next the observer turns around $\pi \mathrm{rad}$. and looks in the opposite direction. He will now characterize the same travelling wave by $(-\lambda,-C)$.

For rotationally symmetric $\beta$ the existence of solutions of (3.8) was studied by Diekmann (1978). He noted that: (i) $L$ is defined in a (right hand) neighbour- 
hood of $\lambda=0$; (ii) for fixed $C, L$ is a convex function of $\lambda$; (iii) for every $\lambda>0$, $L$ is a decreasing function of $C$; and (iv)

$$
\left.\frac{\partial L}{\partial \lambda}\right|_{\lambda=0}<(=) 0 \text { for; } C>(=) 0 .
$$

In particular (iv) implies that one can restrict attention to $C>0$. In the non-rotationally symmetric case (iv) becomes

$$
\frac{\partial L}{\partial \lambda}(0)=R_{0}\left(\bar{\xi}_{1}-C \bar{a}\right)
$$

where

$$
\bar{\xi}_{1}=\int_{0}^{\infty} \int_{-\infty}^{\infty} \xi_{1} \beta\left(a, \xi_{1}\right) d \xi_{1} d a
$$

and

$$
\bar{a}=\int_{0}^{\infty} \int_{-\infty}^{\infty} a \beta\left(a, \xi_{1}\right) d \xi_{1} d a .
$$

Now there can be solutions with $C \leqslant 0$, as we saw in the diffusion equation models. (In Sect. 5 an example is given where we find negative velocities.) In addition to (iii), we note that $L$ is defined for $C \in\left(c_{1}, c_{2}\right)$, where $c_{1} \leqslant 0, c_{2}>c_{1}$ and $c_{1}, c_{2} \in \mathbb{R}$. Furthermore, if $C \downarrow c_{1}, L \rightarrow \infty$, and (iii) holds for every $C \in\left(c_{1}, c_{2}\right)$.

Now, under our assumption $R_{0}>1$ and following the same reasoning as in Diekmann (1978) it can be concluded that there is a $C_{0} \in \mathbb{R}$ such that there exist travelling wave solutions of (3.5) for every $C \geqslant C_{0}$. This $C_{0}$ can be calculated from

$$
\begin{aligned}
L\left(C_{0}, \lambda_{0}\right) & =1, \\
\frac{\partial L}{\partial \lambda}\left(C_{0}, \lambda_{0}\right) & =0 .
\end{aligned}
$$

The asymptotic velocity of population expansion. For rotationally symmetric $\beta$, the $n$-dimensional version of Eq. (3.5) is a special case of the model studied by Diekmann $(1978,1979)$ and Thieme $(1977,1979)$. They proved that if one assumes that the founder population has bounded support, $C_{0}$ is the asymptotic velocity of population expansion in the sense of Eq. (2.3). In an $n$-dimensional discrete time model Weinberger $(1978,1982)$ showed that knowledge of the minimal wave velocity of plane fronts in all directions suffices to describe the asymptotic velocity of spread in the non-rotationally symmetric case as well. (For related work of Lui we refer to Creegan and Lui (1984) and the references given there.) Although a proof for the continuous version of the non-rotationally symmetric case is still lacking, we are convinced that one can safely assume that the minimal wave velocity of plane fronts, $C_{0}$, determines (in a manner described below) the asymptotic velocity of population expansion for the population model (3.5). 
Contours of equal birth rate. As discussed above there is a difference between the minimal velocity, $C_{0}(\psi)$, of a plane wave travelling in a direction which makes an angle $\psi$ with the $x_{1}$-axis, and the asymptotic velocity, $V(\varrho)$ of population expansion along a straight line from the origin making an angle $\varrho$ with the $x_{1}$-axis (Fig. 1). Given $C_{0}(\psi)$, how can we calculate $V(\varrho)$ ? In Appendix I we derive that, under the biologically reasonable assumption that $V$ is a smooth function of $\varrho, V$ and $C_{0}$ are related by

$$
\begin{aligned}
V(\varrho) \cos (\psi-\varrho) & =C_{0}(\psi), \\
-V(\varrho) \sin (\psi-\varrho) & =C_{0}^{\prime}(\psi) .
\end{aligned}
$$

In concrete calculations we may vary $\psi$ from 0 to $2 \pi$ and solve (3.12) for $V$ and $\varrho$. The collection of pairs $(\varrho, V)$ so obtained yields the 'graph' of $V$. This 'graph' is directly related to the contours of equal birth rate. Looking at the population from high up in the air we will see that for sufficiently large $t$ these contours are approximately given by

$$
\{(t V(\varrho) \cos (\rho), t V(\varrho) \sin (\varrho)) \mid \varrho \in[0,2 \pi)\} .
$$

Remark. In his paper on a discrete time model for the spatial spread of genes, Weinberger (1983) also considers the relation between $C_{0}(\psi)$ and $V(\varrho)$. In Appendix I we show how Weinberger's results are related to (3.12).

\section{Non-linear models: How robust are the linear results?}

In Sect. 3 we considered density independent population growth.

Of course at higher densities the population will, through its influence on the environment, which in its turn influences the birth-and-dispersal kernel, change its own dynamical properties. Is the velocity of population expansion in the non-linear case equal to the one in the linear case?

Diekmann $(1978,1979)$ and Thieme $(1977 \mathrm{a}, \mathrm{b} ; 1979 \mathrm{a}, \mathrm{b})$ investigated, for rotationally symmetric dispersal, a special type of non-linearity. In his discrete time model Weinberger (1982) investigated a similar type of non-linearity, and he included non-rotationally symmetric dispersal. Both Diekmann and Thieme and Weinberger showed that the minimal velocity of population expansion of the corresponding linear model is still the asymptotic velocity of population expansion of the full non-linear variant. The particular types of density dependence considered by these authors have clear biological relevance and the results give much insight. One would, however, like to have results on non-linear models where the density dependent feedback is of a more general nature. Such results are absent in the literature.

Together with a redefinition of 'asymptotic velocity of population expansion' we developed, on basis of some worked examples, a conjecture on the velocity of population expansion of non-linear models. These notes were included in the preprint version of this part and they are available on request. 
In loose biological terms the conjecture runs as follows: Consider a nonlinear model for the spatial spread of a population and its associated linear version found by assuming that the population lives forever in a 'virgin' environment.

Assume that:

(i) the average rate of reproduction of an individual experiencing throughout its life an environment 'occupied' by a certain (possibly varying) population is always smaller than the rate of reproduction in a 'virgin' environment (i.e. in particular there are no Allee-like effects), and that

(ii) the influence of an individual on the environment very far from its (present) position is negligible.

Assumption (i) implies that the total birth rate at time $t$ at position $x$ is always smaller in the non-linear model than in the linear variant. From this, one expects that the asymptotic velocity of population expansion of a non-linear model can never be larger than that of its linear variant. Assumption (ii) suggests that the population dynamical behaviour of the forefront of an expanding population is accurately described by the linear model. Thus we expect that the asymptotic velocity of population expansion of such a non-linear model can never be smaller than that of its linear variant.

Together, these observations lead us to conjecture that the asymptotic velocity of population expansion of a non-linear model satisfying (i) and (ii) for the spatial spread of a population equals that of its linear variant.

\section{Some numerical examples}

The general linear model can be adapted to a particular species by an appropriate choice of $\beta(a, x)$. Under the assumption of statistical independence of reproduction and dispersal this amounts to a choice of two functions, $\beta^{0}$ and $D$ (see (3.4)). In this section we introduce some candidates in the form of parametrized families of functions. We use two mechanistic submodels for dispersal together with two descriptive submodels for reproduction. Next we show how $C_{0}$ depends on various parameters.

\subsection{Rotationally symmetric dispersal}

Models for the dispersal density. In the paper 'Models of dispersal in biological systems', Othmer et al. (1988) give an excellent overview of mechanistic models of individual dispersal behaviour. Such models can be used as submodels for the dispersal density, $D$. Here, we will use two mechanistic models for dispersal that lead to well-known probability densities with simple Laplace transforms.

(i) The Gaussian density. This density arises when juveniles move at random till a certain age and then settle down on a permanent breeding ground (e.g. sessile 
aquatic organisms with swimming larvae). It also arises in the case of infectious diseases amongst animals, when transmission is through physical contacts. The relative frequency with which places within a home-range are visited can often be described by a Gaussian density. Now, let two individuals have the centres of their home-ranges at 0 and at $x$ respectively. The probability of encounter per unit of time then is proportional to

$$
\frac{1}{\left(2 \pi \sigma_{1}^{2}\right)^{2}} \int_{\mathbb{R}^{2}} \exp \left(-\frac{|\xi|^{2}}{2 \sigma_{1}^{2}}\right) \exp \left(-\frac{|\xi-x|^{2}}{2 \sigma_{1}^{2}}\right) d \xi=\frac{1}{2 \pi \sigma_{2}^{2}} \exp \left(-\frac{|x|^{2}}{2 \sigma_{2}^{2}}\right)=: D(x)
$$

where $\sigma_{1}^{2}$ is the variance of the Gaussian density describing the relative visiting frequency within the home-range, and $\sigma_{2}^{2}=2 \sigma_{1}^{2}$ is the variance of the Gaussian density describing the contact rate with neighbouring individuals. The marginal distribution is also Gaussian, with variance $\sigma_{2}^{2}$.

(ii) The Bessel density. Assume the juveniles of a species move at random with diffusion constant $s$, and that they settle down at a constant rate $\varphi$. In a slightly different context Williams (1961) and Broadbent and Kendall (1953) showed that these assumptions lead to a distribution of settled individuals

$$
\begin{aligned}
D(x) & =\frac{1}{4 \pi \sigma_{1}^{2}} \int_{0}^{\infty} \frac{1}{\tau} \exp \left[-\tau-\frac{|x|^{2}}{4 \sigma_{1}^{2} \tau}\right] d \tau \\
& =\frac{1}{2 \pi \sigma_{1}^{2}} K_{0}\left(\frac{x}{\sigma_{1}}\right),
\end{aligned}
$$

where $\sigma_{1}^{2}=s / 2 \varphi$ is the variance of the position of settled individuals on a line transect through the source and $K_{0}$ is the modified Bessel function of the second kind of order zero.

The marginal density is the double-exponential density

$$
D(x)=\frac{1}{2} \sqrt{2} \frac{1}{\sigma_{2}} \exp \left[-\sqrt{2} \frac{1}{\sigma_{2}}|x|\right] .
$$

The relation between $\sigma_{1}$ and $\sigma_{2}$ is

$$
2 \sigma_{1}^{2}=\sigma_{2}^{2}
$$

This is also an appropriate model for the dispersal of airborne spores or seeds.

Models for the reproduction kernel

(i) The uniform density is described by

$$
\beta^{0}(a)= \begin{cases}0 & \text { for } 0<a<a_{1} \\ a_{2}^{-1} & \text { for } a_{1} \leqslant a \leqslant a_{1}+a_{2} \\ 0 & \text { for } a>a_{1}+a_{2}\end{cases}
$$

where $a_{1}$ is the duration of the juvenile period, and $a_{2}$ is the duration of the reproductive period. The mean, $\mu$, of this density is $\left(a_{1}+a_{2}\right) / 2$, its variance, $v^{2}$, is $a_{2}^{2} / 12$. 

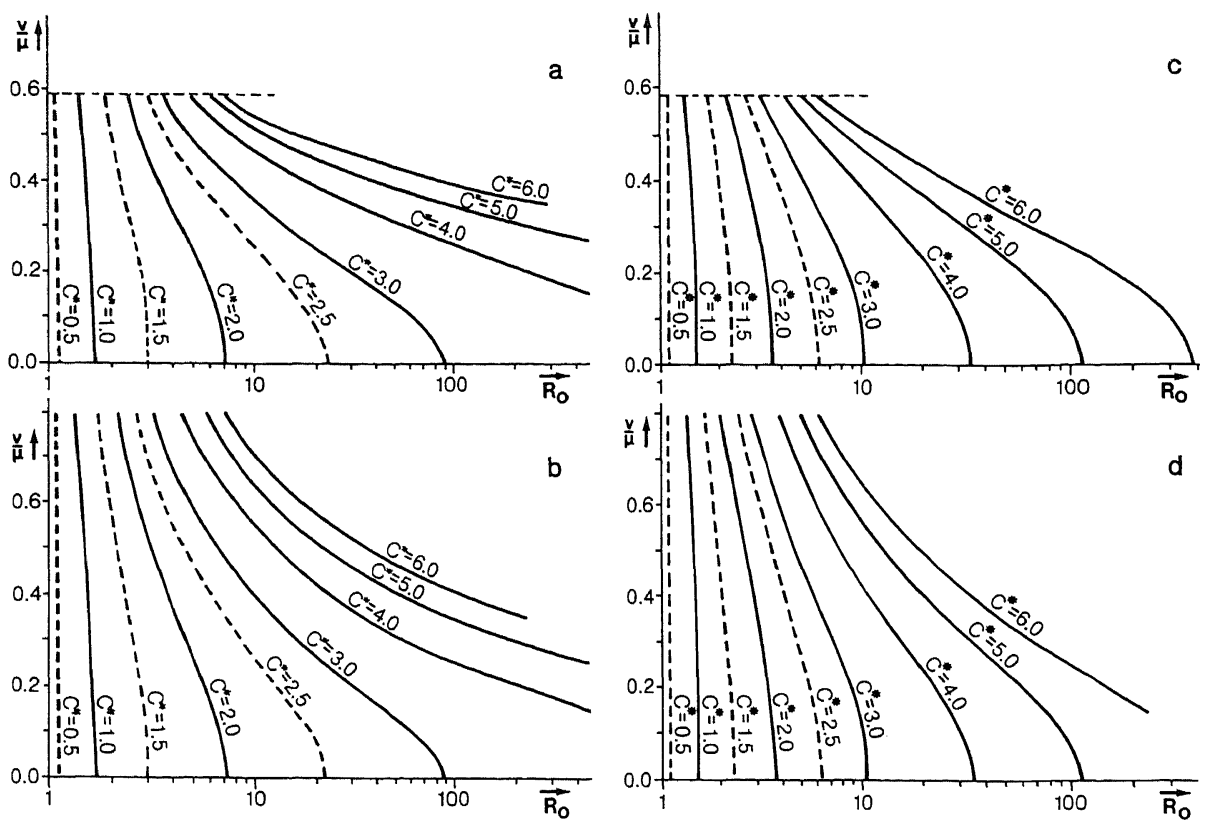

Fig. 3a-d. Contour lines of the scaled wave velocity, $C_{0}^{*}=C_{0} \mu / \sigma$, as a function of the net-reproduction, $R_{0}$, and the coefficient of variation, $v / \mu$, of the reproduction kernel, $\sigma^{2}$ is the variance of the dispersal density. a Uniform density reproduction kernel, (5.5), and Gaussian dispersal density, (5.1). b Gamma-density reproduction kernel, (5.6), and Gaussian dispersal density, (5.1). c Uniform density reproduction kernel, (5.5), and Bessel contact distribution, (5.3). d Gamma-density reproduction kernel, (5.6), and Bessel contact distribution, (5.3)

(ii) The gamma-density

$$
\beta^{0}(a)=\frac{q(q a)^{r-1} \exp (-q a)}{\Gamma(q)} .
$$

The mean, $\mu$, of this density is $r / q$ and its variance, $v^{2}$, is $r / q^{2}$.

Results. Figure 3 shows that the wave velocity increases with increasing $R_{0}$ as is expected. For small $\nu / \mu$, the distance between lines of equal velocity increases with increasing $\ln R_{0}$ in examples with the Gaussian dispersal density, whereas it is constant in examples with the Bessel dispersal density (except near $R_{0}=1$ ). This can be understood by the following heuristic argument. Consider an infinitely long straight line of individuals of age zero. Their contribution to the population density of the next generation at a distance $x$, is roughly proportional to $R_{0} \tilde{D}(x)$, where $\tilde{D}$ is the marginal-density of $D$. Define 'the effective distance', $X_{\text {eff }}$, to be the distance beyond which $R_{0} \tilde{D}(x)$ decreases below a certain number, say $n$. For the Bessel dispersal density

$$
X_{\mathrm{eff}}=\frac{\sigma_{2}}{\sqrt{2}} \ln \left[\frac{R_{0}}{n} \frac{\frac{1}{2} \sqrt{2}}{\sigma_{2}}\right],
$$


which leads us to expect a logarithmic dependence of $C_{0}$ on $R_{0}$. In the case of a Gaussian dispersal density, however,

$$
X_{\text {eff }}=\sigma_{2} \sqrt{2} \sqrt{\ln \left[\frac{R_{0}}{n} \frac{\frac{1}{2} \sqrt{2}}{\sigma_{2} \sqrt{\pi}}\right]},
$$

explaining the observed increases in distance between the lines of equal $C_{0}$ for increasing $R_{0}$.

Another feature, associated with $X_{\text {eff }}$, is that the wave velocities for the Bessel dispersal density are larger than for the Gaussian dispersal density. The wave is dragged forward by the tail of the dispersal density. The tail of the Bessel density is thicker than the tail of the Gaussian density, or in other words if $n>0$, $X_{\text {eff- }}$-Bessel $>X_{\text {eff }}$-Gauss.

When the coefficient of variation of the reproduction kernel is increased, the offspring production will start at a lower age (in the block-function case) or increase faster after $a=0$ (in the gamma-density case). Since offspring that is produced earlier contributes sooner to population growth, the wave velocity becomes larger with increasing coefficient of variation.

It appears that the shape of the dispersal density is more critical to the wave velocity than the shape of the reproduction kernel. We will return to this important point in Sect. 6 and the Discussion.

\subsection{Non-rotationally symmetric dispersal}

The dispersal density. As discussed the Bessel density is a mechanistic model describing the dispersal of spores or seeds under the influence of turbulent diffusion. Often there will also be an average displacement due to a prevailing wind direction. Assume that the wind is blowing in the $x_{2}$-direction; (5.2a) still holds if we put

$$
|x|=\left|\left(x_{1}, x_{2}-m \tau\right)\right|,
$$

where $m$ is the average wind velocity, $m^{*}$, divided by $\varphi$. The marginal density (3.9b) now has the form

$$
\begin{aligned}
\tilde{D}(x)= & \frac{1}{\sqrt{2} \sigma_{2} \sqrt{1+\left(\frac{m}{\sqrt{2} \sigma_{2}}\right)^{2} \sin ^{2} \psi}} \\
& \times \exp \left\{\frac{m}{\sigma_{2}^{2}} \sin \psi x_{1}-\frac{\sqrt{2}}{\sigma_{2}}\left|x_{1}\right| \sqrt{1+\left(\frac{m}{\sqrt{2} \sigma_{2}}\right)^{2} \sin ^{2} \psi}\right\},
\end{aligned}
$$

where $\sigma_{2}$ is given by (5.4).

The reproduction kernel. In this example we use for the reproduction kernel a $\delta$-function at $a=\mu$.

$$
\beta^{0}(a)=\delta(a-\mu)
$$




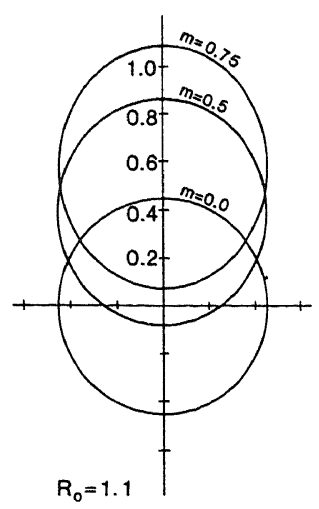

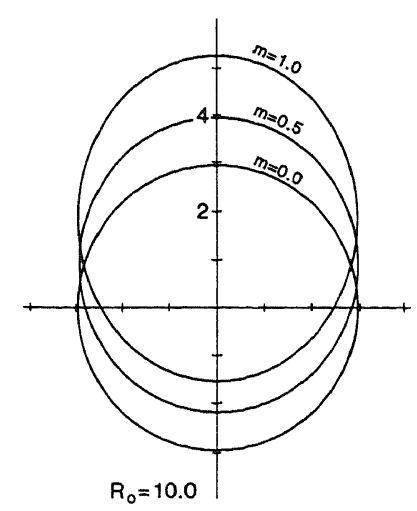

Fig. 4. Contours of equal birth rate for the non-rotationally symmetric dispersal density, (5.10), and a $\delta$ function reproduction kernel, (5.11), for various values of the net-reproduction, $R_{0}$, and wind velocities, $m$

The results can be compared with the results of the rotationally symmetric Bessel density for both reproduction kernels with $v^{2}=0$.

Results. From Fig. 4 we see that for small $R_{0}$ and large $m$ no velocity of population expansion is calculated in the direction opposite to the wind direction. Too few births occur in this direction to build up a population wave and we thus have $V<0$. The contours of equal population density appear to be ellipse shaped. We return to this point in Sect. 6 .

\section{Various approximations}

For every normalized reproduction-and-dispersal kernel $\beta(a, x)$ the associated wave velocity can be calculated, as long as the transform integral (3.9) does exist. It would, however, be useful to have approximations for $C_{0}$ in terms of global characteristics of the normalized reproduction-and-dispersal kernel. Such approximations may provide useful rules of thumb. Moreover, in practice, measurements of the birth kernel have a limited accuracy. This also leads us to search for approximations of $C_{0}$ based on easily measurable quantities. Finally, comparing such approximations with numerical results for specific normalized reproduction-and-dispersal kernels show to what extent $C_{0}$ depends on the details of the reproduction and dispersal process.

\subsection{Rotationally symmetric dispersal}

(i) Small $R_{0}$. Let $l(C, \lambda):=\ln L(C, \lambda)$. Formula (3.9a) then transforms into

$$
l(C, \lambda)=\ln R_{0}+\ln \int_{0}^{\infty} \int_{-\infty}^{\infty} e^{-\lambda(\xi+C a)} \tilde{\beta}(a, \xi) d \xi d a .
$$

The second part of the right-hand side is the so-called cumulant generating function of $\tilde{\beta}$ evaluated at $\lambda$ and $\lambda C$. We shall denote the bivariate cumulants as 
$\kappa_{i j}$ where the first index refers to $\xi$ and the second to $a$. By definition the cumulants are the coefficients in the Taylor expansion of the cumulant generating function. Then (6.1) can be written as

$$
l(C, \lambda)=\ln R_{0}+\sum_{n=1}^{\infty} \sum_{i=1}^{n} \frac{\kappa_{n}-i, i}{(n-i) ! i !} C^{i}(-\lambda)^{n} .
$$

In particular

$$
\kappa_{01}=\mu:=\int_{0}^{\infty} \int_{-\infty}^{\infty} a \tilde{\beta}(a, \xi) d \xi d a,
$$

which is the mean age-at-child-bearing,

$$
\kappa_{02}=v^{2}:=\int_{0}^{\infty} \int_{-\infty}^{\infty} a^{2} \tilde{\beta}(a, \xi) d \xi d a-\mu^{2},
$$

the variance of the age-at-child-bearing,

$$
\kappa_{20}=\sigma^{2}:=\int_{0}^{\infty} \int_{-\infty}^{\infty} \xi^{2} \tilde{\beta}(a, \xi) d \xi d a,
$$

which is the variance of the marginal density of the places where an average female gives birth throughout its life relative to its place of birth. Due to symmetry

$$
\kappa_{i j}=0 \text { for } i \text { odd. }
$$

Further relations between moments and cumulants may be found in Kendall and Stuart (1958). The first terms of system (3.11) become

$$
\left\{\begin{aligned}
0= & \ln R_{0}-\mu C \lambda+\frac{1}{2}\left(\sigma^{2}+v^{2} C^{2}\right) \lambda^{2}-\frac{1}{2} \kappa_{21} C \lambda^{3} \\
& -\frac{1}{6} \kappa_{03} C^{2} \lambda^{3}+\frac{1}{24}\left(\kappa_{40}+6 \kappa_{22} C^{2}+\kappa_{04} C^{4}\right) \lambda^{4}+\cdots, \\
0= & -\mu C+\left(\sigma^{2}+v^{2} C^{2}\right) \lambda-\frac{3}{2} \kappa_{21} \lambda^{2} C-\frac{1}{2} \kappa_{03} C^{3} \lambda^{2} \\
& +\frac{1}{6}\left(\kappa_{40}+6 \kappa_{22} C^{2}+\kappa_{04} C^{4}\right) \lambda^{3}+\cdots
\end{aligned}\right.
$$

Now define $\varepsilon^{2}=\ln R_{0}$, and assume that $C_{0}$ and $\lambda_{0}$ can be written as

$$
\begin{aligned}
& C_{0}=\alpha_{1} \varepsilon+\alpha_{2} \varepsilon^{2}+\alpha_{3} \varepsilon^{3}+\cdots, \\
& \lambda_{0}=\beta_{1} \varepsilon+\beta_{2} \varepsilon^{2}+\beta_{3} \varepsilon^{3}+\cdots .
\end{aligned}
$$

Substitution in (6.3) and solving for subsequent $\alpha_{i}$ 's and $\beta_{i}$ 's leads to

$$
C_{0} \approx \frac{\sigma}{\mu} \sqrt{2 \ln R_{0}}\left[1+\alpha \ln R_{0}\right]
$$

where

$$
\alpha=\left(\frac{\nu}{\mu}\right)^{2}-\kappa_{21} / \sigma^{2} \mu+\frac{1}{12} \kappa_{40} / \sigma^{4}
$$

and

$$
\lambda_{0} \approx \frac{1}{\sigma} \sqrt{2 \ln R_{0}}\left[1-\beta \ln R_{0}\right]
$$


where

$$
\beta=\left(\frac{v}{\mu}\right)^{2}-2 \kappa_{21} / \sigma^{2} \mu+\frac{1}{4} \kappa_{40} / \sigma^{4} .
$$

Note that $\kappa_{40}$ already occurs in $\alpha_{3}$ while $\kappa_{03}$ only occurs in $\alpha_{5}$. This is consistent with the observation in Sect. 4 that the wave velocity is more sensitive to the shape of the dispersal density than to that of the probability density of the age-at-child-bearing.

The wave velocity predicted by approximation formula (6.4) was compared with the values of the four examples from Sect. 4. From Fig. 5 it can be seen that in the case of a Gaussian contact distribution the formula is adequate even when $R_{0}$ is large, provided the coefficient of variation of the probability density of the age-at-child-bearing is small. This is due to the fact that expanding up to the second cumulant amounts to replacing the dispersal density by a Gaussian density. As expected, the parameter area where the approximate formula is accurate is larger for the complete formula (6.4) (expansion up to order $\varepsilon^{2}$ ) than for (6.4) with $\alpha$ set equal to zero (expansion up to order $\varepsilon$ ).
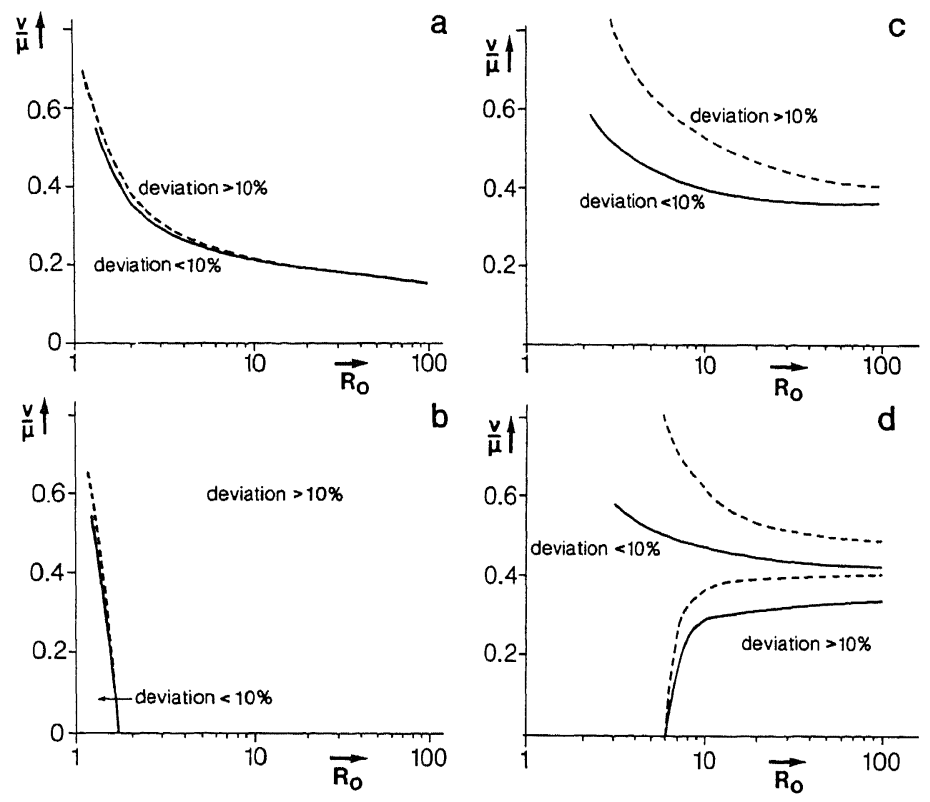

Fig. 5a-d. Parameter region where the wave velocity approximated with (6.4) deviates less than $10 \%$ from the exact value. a Formula (6.4) with $\alpha$ set equal to zero compared to the numerical examples of Fig. 3a,b; b formula (6.4) with $\alpha$ set equal to zero compared to the numerical examples of Fig. $3 \mathrm{c}$,d; $\mathbf{c}$ formula (6.4) compared to the numerical examples of Fig. 3a,b; d formula (6.4) compared to the numerical examples of Fig. 3c,d 
(ii) Concentrated reproduction kernels. The previous expansion is based on the presupposition that $C_{0}$ or, equivalently, $R_{0}-1$ is small. Another possibility is to consider reproduction kernels that are very concentrated. Assume, for the sake of simplicity, that $\tilde{\beta}(a, \xi)=\beta^{0}(a) \tilde{D}(\xi)$, and write

$$
\beta^{0}(a)=\varepsilon^{-1} h\left(\frac{a-\mu}{\varepsilon}\right)
$$

with

$$
\int_{0}^{\infty} a h(a) d a=0 .
$$

Let $\bar{\kappa}_{j}$ denote the $j$ th cumulant of $h$. Then $\kappa_{0 j}=\varepsilon^{j} \bar{\kappa}_{j}$ and $\kappa_{i j}=0$ for both $j \neq 0$ and $i \neq 0$. Breaking off the expansion of $l(C, \lambda)$ after terms with $\varepsilon^{2}$ yields for Gaussian $\tilde{D}$

$$
\begin{gathered}
C_{0}=\frac{\sigma}{\mu} \sqrt{\frac{2 \ln R_{0}}{1-2\left(\frac{v}{\mu}\right)^{2} \ln R_{0}}}, \\
\lambda_{0}=C_{0} \frac{\mu}{\sigma^{2}+\left(\nu C_{0}\right)^{2}} .
\end{gathered}
$$

From Fig. 6 we see that the parameter area where the deviation is smaller than $10 \%$ is slightly larger than the corresponding area for the perturbation expansion (6.4) up to order $\varepsilon$.

When the dispersal density is not Gaussian, the expansion of $l(C, \lambda)$ in general contains infinitely many terms of order one, and we are not able to get a simple explicit expression for $C_{0}$. Yet it may be a good idea, in situations where one has a mechanistic model for the dispersal density, to derive an approximation for $C_{0}$ which is based only on the mean and variance of the reproduction kernel. We shall not elaborate this any further here.

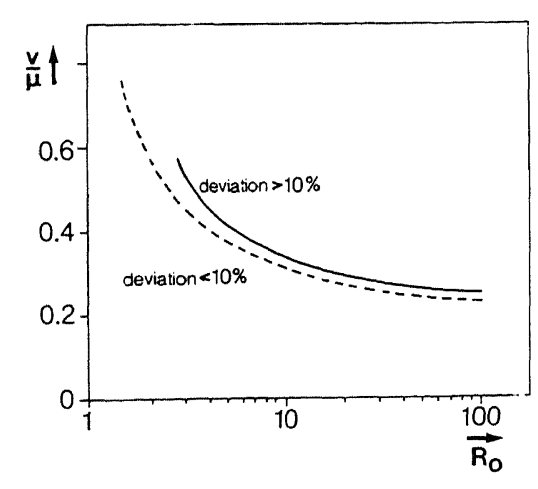

Fig. 6. Parameter region where the wave velocity approximated with (6.6) deviates less than $10 \%$ with the numerical examples of Fig. 3a,b 


\subsection{Non-rotationally symmetric dispersal}

Starting point for the perturbation expansion is again Eq. (6.2). Now the $\kappa_{i j}$ depend on $\psi$, the angle between the direction of movement and the $x_{1}$-axis. Proceeding as before, but taking into account first order terms only, we find

$$
\begin{aligned}
& C_{0}(\psi)=\frac{\kappa_{10}}{\kappa_{01}}+\frac{\sqrt{\kappa_{01}^{2} \kappa_{20}+\kappa_{10}^{2} \kappa_{02}-2 \kappa_{01} \kappa_{10} \kappa_{11}}}{\kappa_{01}^{2}} \sqrt{2 \ln R_{0}}, \\
& \lambda_{0}(\psi)=\left[\kappa_{01}^{2} / \sqrt{\kappa_{01}^{2} \kappa_{20}+\kappa_{10}^{2} \kappa_{02}-2 \kappa_{01} \kappa_{10} \kappa_{11}}\right] \sqrt{2 \ln R_{0}} .
\end{aligned}
$$

The various cumulants now are

(i) $\kappa_{01}=\int_{0}^{\infty} \int_{\mathbb{R}^{2}} a \beta(a, \xi) d a d \xi:=\mu$,

the mean age at child bearing.

(ii) $\kappa_{02}=\int_{0}^{\infty} \int_{\mathbb{R}^{2}} a^{2} \beta(a, \xi) d \xi d a-\mu^{2}:=v^{2}$,

the variance of the age at child bearing.

(iii) $\kappa_{10}=\int_{0}^{\infty} \int_{\mathbb{R}^{2}} \xi \cdot v \beta(a, \xi) d a d \xi:=\gamma_{1} \cos \psi+\gamma_{2} \sin \psi$

where

$$
\gamma_{i}=\int_{-\infty}^{\infty} \xi_{i} \int_{0}^{\infty} \int_{-\infty}^{\infty} \beta(a, \xi) d \xi_{j} d a d \xi_{i} \quad(i, j=1,2 \text { and } i \neq j)
$$

the mean displacement in the $x_{i}$-direction of the birthplace of a child relative to that of its mother.

(iv) $\kappa_{20}=\int_{0}^{\infty} \int_{\mathbb{R}^{2}}\left(\xi \cdot v-\kappa_{10}\right)^{2} \beta(a, \xi) d a d \xi=\sigma_{11}^{2} \cos ^{2} \psi$

$$
+2 \sigma_{12}^{2} \cos \psi \sin \psi+\sigma_{22}^{2} \sin ^{2} \psi
$$

where

$$
\sigma_{i j}^{2}=\int_{0}^{\infty} \int_{\mathbb{R}^{2}}\left(\xi_{i}-\gamma_{i}\right)\left(\xi_{j}-\gamma_{j}\right) \beta(a, \xi) d \xi d a \quad(i, j=1,2)
$$

is the covariance of the components of the displacement in the $x_{1}$-direction

(v) $\kappa_{11}=\int_{0}^{\infty} \int_{\mathbb{R}^{2}}\left(\xi \cdot v-\kappa_{10}\right)(a-\mu) \beta(a, \xi) d \xi d a=\varphi_{1} \cos \psi+\varphi_{2} \sin \psi$

where

$$
\varphi_{1}=\int_{0}^{\infty} \int_{\mathbb{R}^{2}}\left(\xi_{i}-\gamma_{i}\right)(a-\mu) \beta(a, \xi) d \xi d a \quad(i=1,2)
$$


is the covariance of the age at childbearing and the component of the displacement in the $x_{i}$-direction. Substitution into (6.8) and rearranging yields

$$
C_{0}(\psi)=\left(\frac{\gamma_{1}}{\mu} \cos \psi+\frac{\gamma_{2}}{\mu} \sin \psi\right)+\sqrt{2 \frac{\ln R_{0}}{\mu} \cdot P(\psi)}
$$

with

$$
P(\psi)=\left(q_{11} \cos ^{2} \psi+2 q_{12} \cos \psi \sin \psi+q_{22} \sin ^{2} \psi\right) \mu
$$

and

$$
\begin{aligned}
& q_{11}=\left(\mu^{2} \sigma_{11}^{2}+v^{2} \gamma_{1}^{2}-2 \mu \gamma_{1} \varphi_{1}\right) / \mu^{4}, \\
& q_{12}=\left(\mu^{2} \sigma_{12}^{2}+v^{2} \gamma_{1} \gamma_{2}-\mu\left(\gamma_{1} \varphi_{2}+\gamma_{2} \varphi_{1}\right)\right) / \mu^{4}, \\
& q_{22}=\left(\mu^{2} \sigma_{22}^{2}+v^{2} \gamma_{2}^{2}-2 \mu \gamma_{2} \varphi_{2}\right) / \mu^{4} .
\end{aligned}
$$

This completes the derivation of the approximation formula for the velocity of plane wave solutions.

Concerning the contours of equal birth rate and the asymptotic velocity of population expansion in a certain direction from the origin, $V(\varrho)$, we observe that Eq. (6.10a) has the same form as Eq. (2.12). Combining the results of Sect. 2 and formula (6.10) we conclude that, up to the first order in $\varepsilon=\sqrt{\ln R_{0}}, V(\varrho)$ is given by Eq. (2.9) if we replace $m_{1}, m_{2}, f(0), S_{11}, S_{12}, S_{22}$ by $\gamma_{1} / \mu$, $\gamma_{2} / \mu,\left(\ln R_{0}\right) / \mu, \mu q_{11}, \mu q_{12}, \mu q_{22}$ respectively. In Cartesian coordinates the locus of vectors $(V(\varrho) \cos \rho, V(\varrho) \sin \varrho), \varrho \in[0,2 \pi)$, is given by Eq. (2.8) with the same substitution.

\subsection{Connections with the Fisher/Skellam velocity}

Diffusion models are often used in the study of invasions. The most influential model is that of Fisher (1937) and Skellam (1951). In this section we investigate under which conditions the asymptotic wave velocity calculated from such diffusion models is a valid approximation to the asymptotic wave velocity of the general model. We also derive mechanistic interpretations of the phenomenological parameters in these diffusion models.

Randomly moving individuals. One of the basic assumptions of diffusion models is that an individual shows, apart from a systematic movement in a certain direction, random movement. The conditional dispersal density $D^{*}(a, x \mid$ alive $)$, is then given by Eq. (2.7) with $f(0)=0$ and $t$ replaced by $a$. The reproduction-anddispersal kernel is obtained by multiplying $D^{*}$ with the reproduction kernel $\beta^{0}(a)$ defined in Sect. 3.1. Substitution of the resulting kernel in (3.11) yields

$$
\left\{\begin{array}{l}
R_{0} \int_{0}^{\infty} \exp \left\{-a\left(\tilde{P}(\psi) \lambda^{2}+\lambda C^{*}\right)\right\} \beta^{0}(a) d a=1 \\
R_{0}\left[2 \tilde{P}(\psi) \lambda-C^{*}\right] \int_{0}^{\infty} \exp \left\{-a\left(\tilde{P}(\psi) \lambda^{2}+\lambda C^{*}\right)\right\} \beta^{0}(a) d a=0
\end{array}\right.
$$


where

$$
C^{*}=C-m_{1} \cos \psi-m_{2} \sin \psi
$$

and

$$
\tilde{P}(\psi)=s_{11} \cos ^{2} \psi+2 s_{12} \cos \psi \sin \psi+s_{22} \sin ^{2} \psi
$$

The intrinsic rate of natural increase, $r$, can be calculated from the Euler equation (Keyfitz 1968; Roughgarden 1979):

$$
1=R_{0} \int_{0}^{\infty} \exp (-r a) \beta^{0}(a) d a .
$$

Substitution of (6.12) in (6.11) finally yields Eq. (2.12) and

$$
\lambda_{0}(\psi)=\frac{C_{0}-m_{1} \cos \psi-m_{2} \sin \psi}{2 \tilde{P}(\psi)} .
$$

We conclude that provided we identify the intrinsic rate of natural increase with $f(0)$, the wave velocity of the general model and the diffusion model are equal irrespective of the shape of $\beta^{0}(a)$. This justifies the procedure used by, for instance, Lubina and Levin (1988) and Okubo (1986).

Slow growing populations. Individuals of most species do not move at-random throughout their lives. When is the velocity from the diffusion model an approximation to the velocity of population expansion for such species? With the identification

$$
\begin{aligned}
f(0) & =\frac{\ln R_{0}}{\mu}, \\
\tilde{P}(\psi) & =P(\psi) \quad(\text { Eqs. (2.12) and }(6.10 \mathrm{~b})), \\
m_{i} & =\frac{\gamma_{i}}{\mu}, \quad i=1,2,
\end{aligned}
$$

Eq. (2.12) is equal to the perturbation expansion for $C_{0}(\psi)$ (Eq. (6.10)). For the rotationally symmetric case Fig. 5 shows that the diffusion model velocity is a valid approximation when $R_{0}$ is small, say $R_{0} \leqslant 1.5$, i.e. for slow growing populations. Identification (6.13) also relates the phenomenological parameters $f(0), m_{i}$ and $S_{i j}$ to the mechanistic parameters $R_{0}, \mu, v^{2}, \gamma_{i}, \sigma_{i j}$ and $\varphi_{i}$.

Remark 1. The lowest order term in the perturbation expansion of (6.12) reads $r=\ln R_{0} / \mu$ (see Metz and Diekmann 1986, pp. 153-154), which is equal to the identification of $f(0)$ chosen.

Remark 2. On basis of the preceding discussion one might expect that any model for the spatial spread of a population can be approximated by the diffusion model provided that $R_{0}-1 \ll 1$, so that the population grows slowly, and we look at correspondingly large time and space scales. 


\section{Applications}

In this section we give three real-life examples and show how the theory developed in this paper can be put to work.

\subsection{The muskrat (Ondatra zibethicus)}

Observed velocity. As discussed in the introduction, the muskrat started its invasion into Europe in 1905. The velocity of population expansion before 1930 is larger than after 1930 (Hoffman 1958, Fig. 44). This difference is due to the large-scale trapping programs started around 1925/30. The velocity of population expansion in the $1905-1930$ period is $11 \mathrm{~km} \mathrm{year}^{-1}$ (Andow et al., in preparation).

Demography. All available literature data on the life history characteristics of the muskrat are from the period after 1930. Doude van Troostwijk (1976), Becker (1967) and Moens (1978) give age specific survivorship curves, $L(a)$ for field populations. Mallach (1971) calculated that the trapping programs cause about $30 \%$ mortality per year. Using these data $L(a)$ for the $1905-1930$ period is calculated (Table 1).

Approximately $10 \%$ of the young of the year already reproduce in the second half of the breeding season, all individuals reproduce in the spring after their birth (Hoffman 1958; Vincent and Quéré 1972). The number of young per litter is about 6.6, the number of litters per year is approximately 3 (Moens 1978; Vincent and Quéré 1972; Doude van Troostwijk 1976; Hoffman 1958; Artimo 1960). These data provide the age-specific fertility, $m(a)$ (Table 2 ).

The sex-ratio in the muskrat is $0.9: 1.0$ for $q: \sigma^{\lambda}$ (Doude van Troostwijk 1976; Moens 1978; Vincent and Quéré 1972). Together with Table 1 we find $R_{0}=3.1$ (dimensionless) and $\mu=1.41$ (year), $v=0.51$ (year).

Dispersal. The only European large-scale capture-mark-recapture experiment we know is from Mallach (1971). Young of the year as well as the adults disperse. This dispersal mainly takes place between two successive breeding seasons (Verkaik 1987; Mallach 1971). It is well known that males disperse over larger

Table 1. Life-table statistics of the muskrat in the 1905-1930 period

\begin{tabular}{ccccc}
\hline Age interval & $L(a)$ & $m(a)$ & $L(a) m(a)$ & $\beta^{0}(a)$ \\
\hline $0-0.5$ & 0.976 & 0 & 0 & 0 \\
$0.5-1.0$ & 0.788 & 1 & 0.79 & 0.11 \\
$1.0-1.5$ & 0.385 & 10 & 3.85 & 0.56 \\
$1.5-2.0$ & 0.154 & 10 & 1.54 & 0.22 \\
$2.0-2.5$ & 0.060 & 10 & 0.60 & 0.09 \\
$2.5-3.0$ & 0.014 & 10 & 0.14 & 0.02 \\
$3.0-3.5$ & 0.002 & 10 & 0.02 & 0.00 \\
\hline
\end{tabular}


Table 2. The number of recoveries of marked female muskrats, within one year after marking, at various distances from the place of marking (Mallach 1971)

\begin{tabular}{lllllllllllllllllllllllll}
\hline $\begin{array}{l}\text { Class boundary (km) } \\
\text { Number recovered }\end{array}$ & 0 & & 1 & & 2 & & 3 & & 4 & & 5 & & 10 & & 15 & & 20 & & 30 \\
\hline
\end{tabular}

distances than females (Doude van Troostwijk 1976; Mallach 1971; Errington 1963). Successful colonization depends on the dispersal of females. Therefore we use data of Mallach for females recaptured (trapped) within one year after ringing (Table 2).

We thus have information about the distance, $d$, between the capture and recapture position. It can be shown (van den Bosch et al., in preparation) that for $n$ observations of this distance, the variance of the marginal density of recaptured individuals, $\sigma_{\text {catch }}^{2}$, equals

$$
\sigma_{\text {catch }}^{2}=\frac{\sum_{i}\left(d_{i}\right)^{2}}{2 n}
$$

and the kurtosis

$$
\gamma_{\text {catch }}=\frac{1}{\sigma^{4}} \frac{3}{8} \frac{1}{n} \sum_{i}\left(d_{i}\right)^{4}-3
$$

In this case we have information about the recaptured individuals only. So, (7.1) and (7.2) give the parameters of a 'dispersal density of trapped (dead) individuals'. We are, however, interested in the variance and kurtosis of the places where an average individual gives birth during the whole course of its life. It can be shown (van den Bosch et al., in preparation) that these can be calculated from

$$
\sigma^{2}=\frac{\mu}{\mu_{\text {catch }}} \sigma_{\text {catch }}^{2}
$$

and

$$
\gamma=\frac{\mu_{\text {catch }}}{\mu}\left(\gamma_{\text {catch }}-3 \frac{v_{\text {catch }}^{2}}{\mu_{\text {catch }}^{2}}\right)+3\left(\frac{v}{\mu}\right)^{2}
$$

where $\mu_{\text {catch }}$ and $v_{\text {catch }}^{2}$ are the mean age and the variance of the age of the individuals caught in the traps, respectively. Furthermore, in the case of dispersal during the whole course of an individual's life

$$
\frac{\kappa_{21}}{\sigma^{2} \mu}=\left(\frac{v}{\mu}\right)^{2} .
$$

Together with Table 2 we find $\sigma=4.5(\mathrm{~km})$ and $\gamma=4.79$ (dimensionless).

Expected velocity. Substitution of the estimated parameters in (6.4) yields an expected velocity of population expansion of $6.97 \mathrm{~km} \mathrm{year}^{-1}$ for the 19051930 period. 
Discussion. Using data on very accurate controlled experiments on focus expansion Van den Bosch et al. (1988c) found discrepancies of $19 \%$ and $33 \%$ between the observed and expected velocity of focus expansion. In this light and considering the inaccuracy of the type of field data used, a deviation of $36 \%$ between observed and expected velocity of population expansion is reasonable. The data on survival and reproduction stem from growing populations, but whether these populations were still in their exponential phase is questionable. This can cause severe underestimation of the net-reproduction, $R_{0}$. Another major source of error might be in the dispersal density. This density results from only one study. Whether these data are representative for Europe is questionable.

Since the net-reproduction is 3.1 , the Fisher/Skellam velocity is not a valid approximation in this case. Applying the Fisher/Skellam velocity we find $4.8 \mathrm{~km} \mathrm{year}^{-1}$, which deviates $56 \%$ from the observed velocity. This again shows the importance of the higher moments of the dispersal density (cf. Sects. 5 and 6).

Andow et al. (in press) also analyse the muskrat invasion. Much insight in this invasion is gained from their detailed analysis of the velocity of population expansion in various directions. In another paper Andow et al. (in preparation) use the Fisher/Skellam velocity for the muskrat invasion. They estimate the input parameters, $f(0)$, the intrinsic rate of increase and, $s$, the diffusion coefficient, from literature data, and calculate an expected velocity of population expansion between 6 and $32 \mathrm{~km}_{\text {year }}{ }^{-1}$. Using our estimates on $R_{0}$ and $\mu$ and Eq. (6.13) we find $f(0)=0.80$ year $^{-1}$ which is well in the range $0.2-$ 1.1 year $^{-1}$ estimated by Andow et al. From our estimate of $\sigma^{2}$ and (6.13) we find $S=10.24 \mathrm{~km}^{2}$ year ${ }^{-1}$. This is small compared to the range of $5.12-$ $230.1 \mathrm{~km}^{2}$ year $^{-1}$ given by Andow et al. The latter range is calculated from data given by Doude van Troostwijk (1976) and Artimo (1960) on 'distance of solitary muskrats caught, from the nearest pocket of infestation'. There catches comprise both males and females, while, as discussed, males should be left out of the calculations. Moreover, Artimo only gives data for pioneers at large distances from the established population, and gives no notice of pioneers that only travelled short distances. Furthermore, both Doude van Troostwijk and Artimo give no measurements of the time it took the individuals to move the distance given. From this we conclude that the data of Doude van Troostwijk and Artimo are insufficient to calculate parameters of the dispersal. The data from Mallach do not have this problem but yield an estimate of the velocity of population expansion which is too low.

\subsection{Rabies (Lyssavinus spp.)}

Since 1940, a rabies epidemic, originating from Poland, is expanding over Europe. The velocity of expansion ranges from 30 to $60 \mathrm{~km}_{\text {year }}{ }^{-1}$.

Infectivity. Rabies has a long latency period with highly variable duration and a 
short infective period. The infectivity during the infectious period is approximately constant (Sikes 1962). The average duration of the latency and infectious periods is approximately 35 and 5 days, respectively (Berger 1976; MacDonald and Bacon 1982; Bacon 1985). If for the time being we consider $\beta^{\circ}(a)$ to be a uniform distribution then $\mu$ equals approximately 0.1 year. This value of $\mu$ is given by many authors (Anderson 1982; Smith 1985; Källén et al. 1985).

Although there seems to be consensus about the averages, very little information is available about the variability of the latency and the infective period. For the use in his stochastic simulation model, Berger (1976) constructed probability densities for the latency and infectious period based on a small number of experiments done in the thirties by Prof. Dr. G. Schoop (Berger, personal communication). Although these densities might still be far from realistic they at least give some insight. Now consider $\beta^{0}(a)$ to be a block function with a stochastic latency period, $\ell$, and a stochastic infectious period, $i$. In Appendix II it is shown how $\mu$ and $v$, can be calculated for such a stochastic block function. For Berger's data

$$
\begin{gathered}
\mu=33.44 \text { days, } \\
v=4.94 \text { days. }
\end{gathered}
$$

Since the coefficient of variation and $R_{0}$ are both small this term is negligible in (6.4). So, our first conclusion is that detailed information about the probability density of $\ell$ and $i$ is not necessary as long as $\mu$ is accurately determined. In the rest of our discussion we leave the coefficient of variation term out of consideration.

Dispersal. Andrall et al. (1982) reported that three radio-tracked foxes used the same home-range before and during the period in which they were rabid. The only behavioural change appeared to be that the proportion of time spent active increased (thereby increasing the contact rate with other foxes). Therefore 'dispersal' and infectivity production are statistically independent (Eq. (3.4)). Consequently $\kappa_{21}$ equals zero in (6.4).

The frequency with which places in the home-range are visited is well described by a two-dimensional Gaussian density (e.g. Ball 1981). The fourth cumulant of a Gaussian density is zero. We are thus in a situation, where $\alpha=0$ in (6.4). This implies that (6.4) effectively gives the Fisher/Skellam velocity. When space is completely 'filled-up' with fox home-ranges

$$
\sigma_{1}^{2}=\omega F^{-1}
$$

where $\sigma_{1}^{2}$ is the variance of the Gaussian density, $F$ the fox population density and $\omega$ a constant. Lambinet et al. (1978) give

$$
\sigma_{1}=2.3 \mathrm{~km}
$$


at a population density of one fox $\mathrm{km}^{-2}$. So,

$$
\omega \approx 5.3 \text {. }
$$

Using (5.1) we find that the standard deviation, $\sigma_{2}$, of the marginal dispersal density is given by

$$
\sigma_{2}=\sqrt{10.6 F^{-1}}
$$

Net-reproduction. Finally, we need information about the net-reproduction, $R_{0}$. It is obvious that $R_{0}$ depends on population density. We make the assumption that

$$
R_{0}=\Theta F
$$

The threshold population density, $F_{T}$, below which no rabies epidemic is possible is reported to be in the range 0.25 to 1.0 fox $\mathrm{km}^{-2}$ (Lloyd 1976; Andral and Toma 1977). The most recent, and therefore hopefully most accurate, estimate of $F_{T}$ is $0.4 \mathrm{fox} \mathrm{km}^{-2}$ (Steck and Wandeler 1980). At this threshold $R_{0}=1$, so

$$
\Theta=2.5 \mathrm{~km}^{2} \text { fox }{ }^{-1} \text {. }
$$

Velocity of disease expansion. Substitution of (7.6) and (7.7) in (6.4) yields

$$
C_{0}=2 \frac{\sqrt{\omega}}{\mu} \sqrt{\frac{\ln (\Theta F)}{F}} .
$$

The resulting relations between $C_{0}$ and $F$ is depicted in Fig. 7.

Discussion. A striking feature is that intermediate velocities only occur for extremely small ranges of fox density. There is either an epidemic travelling at a, more or less, constant velocity, or there is no epidemic at all. This corresponds to observations in the field (e.g. Bögel and Moegle 1980). Another remarkable thing is that for somewhat larger densities the velocity of rabies expansion slightly decreases with increasing fox population density. We do not know of any data confirming or falsifying this.

Källén et al. (1985) also investigate the spatial spread of rabies. They use a diffusion type model and derive a velocity equation which is basically the same as the Fisher/Skellam velocity. The (major) difference between their and our

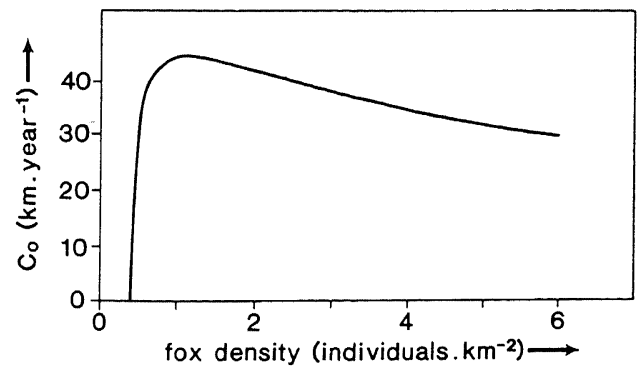

Fig. 7. The velocity of expansion of a rabies epidemic as a function of fox population density 
approach is in the determination of the parameters. In our terminology Källén et al. use

$$
f(0)=\frac{\Theta F-1}{\mu}
$$

which is for small $F$ approximately equal to $\ln (\Theta F) / \mu$ used in (7.8). In order to estimate the diffusion coefficient $s$ Källén et al. assume that when rabies viruses 'enter the limbic system foxes lose their sense of direction and territorial behaviour and start to wander around in a more or less random way'. This is in contradiction to the observations of Andral et al. (1982). Using this assumption $s$ is estimated from $s=K A$ where $A$ is the average territory area and $1 / K$ is the average time after infection until a fox leaves its territory. A mechanistic basis for this estimation is not given. The spatial behaviour of foxes is so widely different from the continuous random movement incorporated in the diffusion model that the interpretation and estimation of $s$ creates a major difficulty. This results in the unconvincing estimation method described. Using the identification (6.13) overcomes this problem. Due to the absence of an influence of fox density on territory size, the velocity of rabies spread calculated by Källén et al. increases with population density while equation (7.8) predicts a slightly decreasing $C_{0}$ at the somewhat larger population densities. It would be worthwhile to confront these two opposite predictions with field data.

\subsection{Focus development in cultivar mixtures}

Many fungal plant diseases develop so-called foci or hot-spots. Disease foci originate from a single infected plant. From this centre of infection the disease expands in a circular pattern. Van den Bosch et al. (1988a,b,c) used the Diekmann/Thieme model to study the radial velocity of focus expansion. We refer to these papers for further information. Here we only illustrate how the model can be used to gain insight in possible strategies for disease control. A more detailed presentation of the experiment presented here, will be published elsewhere (van den Bosch et al., in press).

Motivation. The effectiveness of cultivar mixtures to control fungal diseases of the foliage in small grain corps is well documented (e.g. Browning and Frey 1969; Wolfe 1985). We investigate the velocity of focus expansion in mixtures of susceptible and resistant plants, at constant total plant density.

Theory. Fungal plant diseases are transferred between hosts by means of airborne spores. Inside the canopy layer spores move under the influence of turbulence and are trapped by infected and susceptible plants alike. The transfer mechanism, therefore, satisfies the assumptions underlying the Bessel density (5.2). The discussion of the numerical examples and Eq. (5.7) imply that in this situation

$$
C_{0}=A_{1} \ln R_{0}+B_{1}
$$

where $A_{1}$ and $B_{1}$ are constants. 


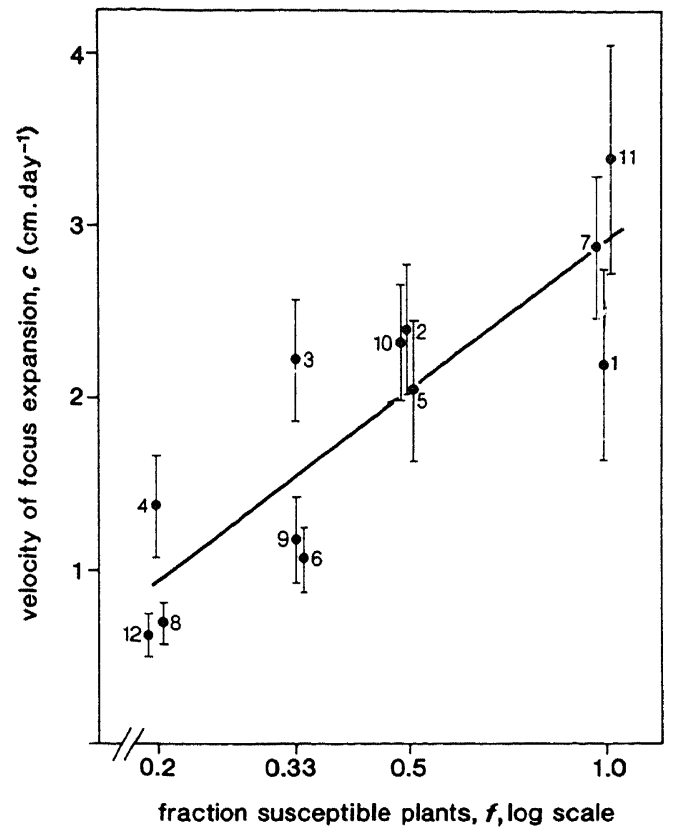

Fig. 8. The velocity of focus expansion, $c$, as a function of the fraction susceptible plants, $f$, in the experimental plots. Points represent observed $c$ values, vertical bars their standard deviations. Entries are plot numbers. The drawn lines is the fitted equation (7.3)

Denote by $\tilde{R}_{0}$ the net-reproduction of the fungus in a field consisting of plants of the susceptible cultivar only. Assume that the plants of the susceptible and resistant cultivar have the same morphology, the same development rate, the same spore trapping characteristics and so on. Then the only effect of the cultivar mixture is that a fraction $1-f$ of the spores is 'removed' from the epidemic process. So,

$$
R_{0}=f \tilde{R}_{0}
$$

Substitution of (7.10) in (7.9) shows that, according to the theory,

$$
C_{0}=A \ln f+B \text {. }
$$

Experiment. A field experiment was designed to test relation (7.11) using the stripe rust (Puccinia striiformis West.)-wheat (Triticum aestivum L.) pathosystem.

Twelve wheat plots were established with mixtures of a susceptible cultivar (Okapi) and a resistant cultivar (Sarno). Mixing ratios 1:0;1:1; 1:2 and 1:4 (Okapi:Sarno) were planted in three replicates. Foci were initiated by planting pots with infected wheat plants at the centre of each plot. The expansion of the focus was assessed at about weekly intervals.

Discussion. The radial velocity of focus expansion shows considerable variation within and between plots (Fig. 8). Equation (7.11), however, fits the data well. Generalizing from the theoretical considerations and the experimental evidence we can state: 
In a mixture of susceptible and resistant plants, the radial velocity of focus expansion increases linearly with the logarithm of the proportion of susceptible plants.

\section{Discussion}

We have shown how the velocity of population expansion can be calculated from knowledge of the demographic and dispersal attributes of individuals. The operationalization of the existing theory, discussed in this paper, also led to several biologically motivated extensions which are now in need of a mathematical analysis.

The asymptotic result from Sect. 3 gives us the velocity with which a population will eventually expand. It does not tell how quickly this asymptote is approached. Simulation models of invasions usually show a rapid convergence to the 'final' velocity (Zadoks and Kampmeyer 1977; Nobel 1974; Ammerman and CavaliZforza 1984; Zawolek 1989; Minogue and Fry 1983a,b). Furthermore many real-life examples also show convergence to a constant velocity within the time span of the invasion (Hengeveld 1989; Andow et al., in press; Okubo 1988; Van den Bosch 1988c; Minogue and Fry 1983). Although it would be useful to have estimates on the speed of convergence, we have a gut feeling that at least the front of invading populations approaches the asymptotic velocity within an 'experimentally' reasonable time span and that the asymptotic result of Sect. 3 can be used in many situations.

In Sects. 5 and 6 we showed that the shape of the dispersal density is a major determinant of the asymptotic velocity of population expansion. In experimental and data analytical papers on population expansion it often turns out that data on the dispersal of individuals around their place of birth are difficult to assess and often scarce or absent. Much experimental effort is needed to improve this situation. Besides the estimation of 'distance dispersed', much effort should be given to the disperal behaviour itself since this behaviour determines the shape of the disperal density. For this kind of research the paper by Othmer et al. (1988) presents a perfect guideline.

The advantage of the present approach, based on the work of Diekmann and Thieme, is that, contrary to diffusion models, not many assumptions are made about reproduction, survival and dispersal of individuals. Amongst the only assumptions made are (i) the absence of Allee like effects and (ii) the presence of a sufficiently large range of population densities between the very low densities where demographic stochasticity becomes apparent, and the large densities where non-linear effects raise their head. (See Mollison $(1985,1986)$ for a discussion of the consequences of this last assumption being flouted.) This general approach yields a correspondingly general result about the velocity of population expansion. The real-life examples discussed in Sect. 7 furthermore show that we have been able to catch the essentials of the processes underlying population expansion for a wide variety of species. Consequently, analyzing the velocity aspect of invasions is open. Such studies are expected to give insight into the important ecological phenomenon of biological invasions. 
Acknowledgements. We thank H. R. Thieme for organizing the Heidelberg workshop on the mathematical modelling of epidemics in November 1979. The present paper is a direct offshoot of this workshop. We are also grateful to Y. Zitman and H. Regeer for typing the manuscript and to G. P. G. Hock for drawing the figures, and to the coauthors of our more experimentally oriented papers, J. C. Zadoks and R. Hengeveld, for discussions about the experimental application of the theoretical work developed in this paper.

\section{Appendix I: The relation between $V(\varrho)$ and $C_{0}(\psi)$}

Let $V(\varrho)$ be the asymptotic velocity of population expansion in the $\varrho$ direction, i.e.

$$
\lim _{t \rightarrow \infty} b(t, t y)= \begin{cases}0 & \text { for all } y \text { outside } A \\ \infty & \text { for all } y \text { inside } A\end{cases}
$$

where

$$
A=\left\{V(\varrho)\left(\begin{array}{c}
\cos \varrho \\
\sin \varrho
\end{array}\right): \varrho \in[0,2 \pi)\right\} .
$$

Let $C_{0}(\psi)$ be the minimal wave speed in the direction $\psi$. We expect that a consistency relation between $V(\varrho)$ and $C_{0}(\psi)$ must hold. We first present a geometrical derivation of this relation and then turn to a result of Weinberger (1983).

Assume that $V(\varrho)$ is a smooth function of $\varrho$. Now, consider Fig. 9. Translate the intersection point $(x, y)$ to the origin and rotate the figure over $\frac{1}{2} \pi-\psi$. The contour can, due to our smoothness assumption, locally be approximated by

$$
y=-a x^{2}+o\left(x^{2}\right) .
$$

Now let $\Theta(x)$ be the angle with the $x$-axis of the normal to the contour at point $x$ (Fig. 9b). Then

$$
\Theta=\frac{1}{2} \pi+\arctan (-2 a x)=\frac{1}{2} \pi-2 a x+O\left(x^{3}\right) .
$$

In a small timestep $\Delta$ the contour is at most $O(\Delta)$ translated, so we can restrict

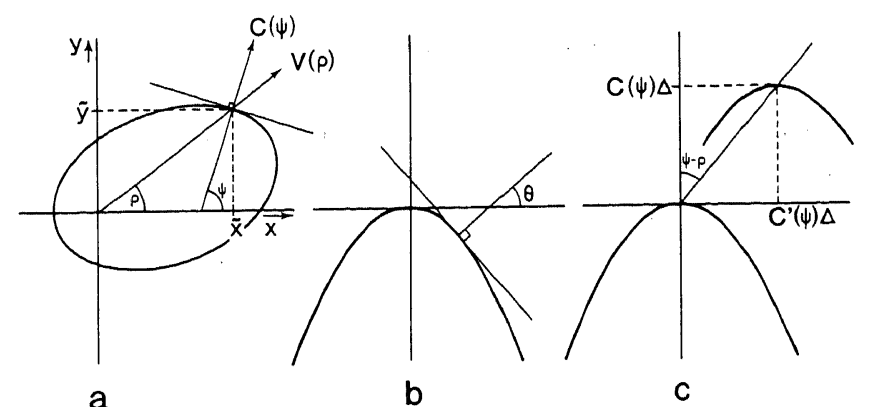

Fig. 9. See the text 
to $x$ 's of this order. In the timestep $\Delta$ the point $(x, y)$ on the contour is translated to:

$$
\begin{aligned}
(\bar{x}, \bar{y}) & =\left(x+C\left(\psi+\Theta-\frac{\pi}{2}\right) \cos (\Theta) \Delta ; y+C\left(\psi+\Theta-\frac{\pi}{2}\right) \sin (\Theta) \Delta\right) \\
& \approx(x+C(\psi-2 a x) 2 a x \Delta ; y+C(\psi-2 a x) \Delta) .
\end{aligned}
$$

So, the translated contour is up to second order terms given by

$$
y=-a x^{2}+C(\psi-2 a x) \Delta \approx-a x^{2}-C^{\prime}(\psi) 2 a x \Delta+C(\psi) \Delta
$$

(see Fig. 9c), and the new top of the parabola is now

$$
(\bar{x}, \bar{y})=\left(-C^{\prime}(\psi) \Delta ; C(\psi) \Delta\right)
$$

which finally leads to

$$
\begin{gathered}
\psi-\varrho=\arctan \left(-\frac{C^{\prime}(\psi)}{C(\psi)}\right) \\
V(\varrho)=\sqrt{(C(\psi))^{2}+\left(C^{\prime}(\psi)\right)^{2}}
\end{gathered}
$$

or equivalently Eq. (3.12).

Weinberger (1983) has derived the characterization

$$
A=\left\{x: x_{1} \cos \psi+x_{2} \sin \psi \leqslant C_{0}(\psi) \text { for all } \psi \in[0,2 \pi)\right\}
$$

in the context of a discrete time model. Combining (I.2) and (I.3) we find

$$
V(\varrho) \leqslant \frac{C_{0}(\psi)}{\cos (\psi-\varrho)}:=F_{\varrho}(\psi), \text { for all } \psi \in[0,2 \pi) \text {. }
$$

A point $x$ of the boundary separating the 'inside' from the 'outside' of the set $A$ necessarily satisfies

or similarly

$$
V(\varrho)=\min _{\psi} F_{\varrho}(\psi)
$$

$$
\frac{d F_{Q}}{d \psi}=0 ; \quad V(\varrho)=F_{\varrho}(\psi)
$$

which, in its turn, equals (3.12).

Inequality (I.4) is more restrictive than (I.5). What then is the difference between (3.12) and the Weinberger result? To answer this question consider the function $F_{e}(\psi)$. Note that for $(\psi-\varrho) \rightarrow \frac{1}{2} \pi, F_{\varrho}(\psi) \rightarrow \infty$. Using the first equation of (I.6) we possibly find, besides the absolute minimum, one or more local minima and maxima. If such a local minimum exist it can if we gradually change $\varrho$ : (a) disappear because the local minimum and maximum fuse, (b) become the absolute minimum leaving the former absolute minimum as local minimum, or (c) a combination of these two. Combining this with the second equation of (I.6) 
it can be seen that the graph of $V(\varrho)$ consists, at least, of a closed curve resulting from the absolute minimum (the AMCC). The local minima/maxima could possibly cause isolated closed curves 'outside' the AMCC, loops on the 'outside' of the AMCC fusing with the AMCC at one point, or more exotic possibilities like double loops in conjunction with closed curves, etc. The important point is that the properties of $F_{Q}(\psi)$ mentioned ensure that loops and isolated closed curves cannot occur 'inside' the AMCC. The closed convex set $A$ as given by Weinberger equals the AMCC leaving our the isolated closed curves and pruning the loops. This pruning may result in a sharp angle. It is at such sharp angles where the geometrical derivation breaks down.

In conclusion we can say that Eq. (3.12) tells the whole story if $V(\varrho)$ is a smooth function of $\varrho$, which is biologically reasonable. In other situations (3.12) also gives us the desired contour of equal birth rate/population density after pruning of loops and crossing out isolated islands. Whether or not such loops or islands do actually occur for 'decent' kernels we do not know.

\section{Appendix II: The mean and variance of a stochastic block function}

In this appendix we show how the mean and variance of the reproduction kernel can be calculated for a stochastic block function. Since these calculations are used in the rabies example we use the terminology of infectious diseases.

Consider a block function with a stochastic latency period $(\underline{\ell})$, a stochastic infective period $(i)$ and a stochastic infectiousness ( $\underline{\zeta}$. When $\underline{\ell}$ and $\underline{i}$ are independent variables, respectively with probability density $f_{\ell}$ and survivor function $\mathscr{H}_{i}$, the average infectiousness of an individual $\tau$ time after infection is

$$
i(\tau)=(\mathscr{E}[\underline{i}])^{-1} \int_{0}^{\tau} f(x) \mathscr{H}_{i}(\tau-x) d x .
$$

This model subsumes the usual two stages (latent and infective) differential equation models in which both $f_{l}$ and $\mathscr{H}_{i}$ are assumed to be exponential. The two special time kernels from Sect. 4, the block and gamma kernels, can be derived from it by assuming the densities of $i$ and $\ell$ to be respectively both degenerated and gamma $(\varrho-1, \ell)$ and exponential $(\ell)$.

The mean and variance of $i(\tau)$ can be calculated as follows. The mean infectivity of an individual is given by

$$
I(\tau)=\mathscr{E}[\eta(\tau)],
$$

where the individual infectivity $\underline{\eta}$ equals

$$
\eta(\tau)=\underline{\zeta}\{H(\tau-\underline{\ell})-H(\tau-\underline{\ell}-\underline{i})\}
$$

with $H$ the Heaviside step-function. And

$$
\gamma=\int_{0}^{\infty} I(\tau) d \tau=\mathscr{E}\left[\int_{\underline{\ell}}^{\underline{\ell}+\underline{i}} n(\tau) D \tau\right]=\mathscr{E}[\underline{\zeta i}] .
$$


By definition $i(\tau)=\ell^{-1} / \gamma I(\tau)$. The mean, $\mu$, of the time kernel is found from

$$
\mu=\int_{0}^{\infty} \tau i(\tau) d \tau=\gamma^{-1}\left[\int_{\underline{\ell}}^{\underline{\ell}+\underline{i}} \tau \underline{\eta}(\tau) d \tau\right]=\frac{\mathscr{E}\left[\underline{i \ell \zeta]}+\frac{1}{2}\left[\underline{\left.\zeta i^{2}\right]}\right.\right.}{\mathscr{E}[\underline{\zeta i}]} .
$$

When $\underline{\ell}, \underline{i}$ and $\underline{\zeta}$ are independent

$$
\mu=\mathscr{E}[\ell]+\frac{1}{2} \mathscr{E}[\underline{i}]\left(1+C^{2}[\underline{i}]\right),
$$

where $C[i]$ is the coefficient of variation of $i$. The calculation of the variance, $v^{2}$, proceeds along the same lines

$$
\mu^{2}+v^{2}=\int_{0}^{\infty} \tau^{2} I(\tau) d \tau=\gamma^{-1} \mathscr{E}\left[\int_{\underline{\ell}}^{\underline{\ell}+\underline{i}} \tau^{2} \underline{\eta}(\tau) d \tau\right]=\frac{\mathscr{E}\left[\underline{\zeta \ell^{2}} \underline{i}\right]+\left[\underline{\zeta \ell i^{2}}\right]+\frac{1}{3} \mathscr{E}\left[\underline{\zeta i^{3}}\right]}{\mathscr{E}[\underline{\zeta i}]}
$$

And under the independence assumption,

$$
v^{2}=\operatorname{var}[\underline{\ell}]+\frac{1}{2} \operatorname{var}[\underline{i}]\left(1-\frac{1}{2} C^{2}[\underline{i}]\right)+(\mathscr{E}[\underline{i}])^{2}\left(\frac{1}{12}+\frac{1}{3} S[\underline{i}] C^{3}[\underline{i}]\right),
$$

where $S[i]$ is the skewness of $i$.

(II.1) and (II.2) relate the quantities used in Sect. 5 to quantities which are sometimes slightly better known. Usually we can make fair guesses about the mean and variance of the latency period. (II.1) and (II.2) moreover show that contrary to the situation for the contact distribution in the case of the time kernel, an approach based on a direct phenomenological measurement may be preferable to an approach based on detailed micromodels. A quantity like the skewness of the infective period, $S[i]$, is difficult to measure. Yet this quantity occurs already in the expression for the variance, $v^{2}$, of the time kernel.

For the probability density given by Berger (1976) we find

$$
\begin{array}{cll}
C(\underline{i})=0.32 ; & \mathscr{E}(\underline{\ell})=2.99 ; & \mathscr{E}(\underline{i})=7.9 ; \\
\operatorname{var}(\underline{\ell})=14.08 ; & \operatorname{var}(\underline{i})=6.47 ; & S(\underline{i})=2.96 .
\end{array}
$$

\section{References}

Ammerman, A. J., Cavalli-Sforza, L. L.: The neolithic transition and the genetics of populations in Europe. Princeton University Press 1984

Anderson, R. M. (ed.): Population dynamics of infectious diseases. Theory and applications. London: Chapman and Hall 1982

Andow, D. A., Kareiva, P. M., Levin, S. A., Okubo, A.: Spread of invading organisms: patterns of spread. In: Kim, K. C. (ed.) Evolution of insect pests: the pattern of variations. New York: Wiley

Andow, D. A., Kareiva, P. M., Levin, S. A., Okubo, A.: Spread of invading organisms, submitted Andral, L., Artois, M., Aubert, M. F. A., Blancou, J.: Radio-pistage de renards enrages. Comp. Immunol. Microbiol. Infect. Diseases 5, 284-291 (1982)

Andral, L., Toma, B.: La rage en France en 1976. Rec. Med. vet. 153, 503-508 (1977)

Anonymous: Ecology of biological invasions. SCOPE Newsletter 23, 1-5 (1985)

Aronson, D. G., Weinberger, H. F.: Nonlinear diffusion in population genetics, combustion, and nerve pulse propagation. In: Goldstein, J. A. (ed.) Partial differential equations and related topics. (Lect. Notes Math., vol. 446, pp. 5-49) Berlin Heidelberg New York: Springer 1975 
Aronson, D. G., Weinberger, H. F.: Multidimensional nonlinear diffusion arising in population genetics. Adv. Math. 30, 33-76 (1978)

Artimo, A.: The dispersal and the acclimatisation of the muskrat Ondatra zibetica (L.), in Finland. Papers on game research 21, 1-101 (1960)

Bacon, P. J. (ed.): Population dynamics of rabies in wildlife. New York: Academic Press 1985

Ball, F. G.: Some statistical problems in the epidemiology of fox rabies. Thesis 1981, University of Nottingham

Becker, K.: Populationsstudien an Bismratten (Ondatra zibethica L.) I Zoologische Beiträge 13, 369-396 (1967)

Berger, J.: Model of rabies control. In: Berger, J., Buhler, W., Repges, R., Tautu, P. (eds.) Mathematical models in medicine. (Lect. Notes Biomath., vol. 11, pp. 75-88) Berlin Heidelberg New York: Springer 1976

Bögel, K., Moegle, H.: Characteristics of the spread of a wildlife rabies epidemic in Europe. Biogeographica 8, 251-258 (1980)

Bramson, M.: Convergence of solutions of the Kolmogorov equation to travelling waves. Mem. Am. Math. Soc. 44, 190 (1983)

Broadbent, S. R., Kendall, D. G.: The random walk of Trichostrongylus retortaeformis. Biometrika 9, 460-465 (1953)

Browning, J. A., Frey, K. J.: Multiline cultivars as a means of disease control. Annu. Rev. Phytopathol. 7, 355-382 (1969)

Caughley, G.: Liberation, dispersal and distribution of Himalayas Thar (Hemitragus jemlahicus) in New Zealand. New Zealand J. Sci. 13, 220-239 (1970)

Creegan, P., Lui, R.: Some ramarks about the wave speed and travelling wave solutions of a nonlinear integral generator. J. Math. Biol. 20, 59-68 (1984)

Diekmann, O.: Thresholds and travelling waves for the geographical spread of infection. J. Math. Biol. 6, 109-130 (1978)

Diekmann, O.: Run for your life. A note on the asymptotic speed of propagation of an epidemic. J. Differ. Equations 33, 58-73 (1979)

Diekmann, O.: Dynamics in biomathematical perspective. In: Hazewinkel, M., Lenstra, J. K., Meertens, L. G. L. (eds.) Mathematics and computer sicence II. (CWI Monographs vol. 4, pp. 23-50) 1986

Diekmann, O., Temme, N. M.: Nonlinear diffusion problems. Amsterdam: Mathematical Centre 1976

Doude van Troostwijk, W. J.: The muskrat (Ondatra zibethicus L.) in the Netherlands, its ecological aspects and their consequences for man. Thesis, State University of Leiden

Errington, P. L.: Muskrat populations. Iowa: Iowa State University

Fisher, R. A.: The wave of advance of advantageous genes. Ann. Eugen. 7, 355-369 (1937)

Hadeler, K. P., Rothe, F.: Travelling fronts in nonlinear diffusion equations. J. Math. Biol. 2, 251-263 (1975)

Hengeveld, R.: Dynamics of biological invasions. London: Chapman and Hall 1989

Hoffman, M.: Die Bisamratte. Leipzig: Academische Verlagsgesellschaft 1958

Källen, A., Arcuri, P., Murray, J. D.: A simple model for the spatial spread and control of rabies. J. Theor. Biol. 116, 377-393 (1985)

Kendall, M. G., Stuart, A.: The advanced theory of statistics, vol. I. London: Griffin 1958

Kendall, D. G.: Mathematical models of the spread of infection. In: Mathematics and computer sicence in biology and medicine (Medical Research Council, London, pp. 213-224) 1965

Keyfitz, N.: Introduction to the mathematics of population. Reading, Mass.: Addison Wesley 1968

Kolmogorov, A., Petrovsky, I., Piscounov, N.: Etude de l'équation de la diffusion avec croissance de la quantité de matiere et son application a un probleme biologique. Mosc, Univ. Math. Bull. 1, $1-25$ (1937)

Kornberg, H., Williamson, M. H.: Quantitative aspects of the ecology of biological invasions. London: Royal Society, 1987

Lambinet, D., Boisvieux, J. F., Mallet, A., Artois, M., Andral, L.: Modele mathématique de la propagation d'une épizootie de rage vulpine. Rev. Epidém. et Santé Publ. 26, 9-28 (1978)

Levin, S. A.: Analysis of risk for invasions and control programs. In: Drake, J., Castri, F. di, 
Groves, R., Kruger, F., Mooney, H., Rejamenk, M., Williamson, M. (eds.) Biological invasions: a global perspective. Chichester: Wiley, in press

Lloyd, H. G.: Wildlife rabies in Europe and the British situation. Trans. R. Soc. Trop. Med. Hyg. 70, $179-187$ (1976)

Lubina, J. A., Levin, S. A.: The spread of a reinvading species: Range expansion in the California Sea Otter. Am. Nat. 131, 526-543 (1988)

MacDonald, D. W.: Rabies and wildlife. Oxford: Oxford University Press 1980

MacDonald, D. W., Bacon, P. J.: Fox society, contact rate and rabies epizootiology. Comp. Immunol. Microbiol. Infect. Dis. 5, 247-256 (1982)

Mallach, N.: Markierungsversuche zur Analyse des Aktionsraums und der Ortsbewegungen des Bisams (Ondatra zibethica L.) Anzeiger für Schädlingskunde und Pflanzenschutz XLIV9, 129136 (1971)

Metz, J. A. J., Diekmann, O.: The dynamics of physiologically structured populations. (Lect. Notes Biomath., vol. 68) Berlin Heidelberg New York: Springer 1986

Minogue, K. P., Frey, W. E.: Models for the spread of disease: model description. Phytopathology 73, $1168-1173(1983 a)$

Minogue, K. P., Frey, W. E.: Models for the spread of plant disease: some experimental results. Phytophathology 73, 1173-1176 (1983b)

Moens, R.: Etude bio-écologique du rat musqué en Belgique. Parasitica 34, 57-121 (1978)

Mollison, D.: The rate of spatial propagation of simple epidemics. In: Le Cam, L. M., Neyman, J., Scott, E. L. (eds.) Proc. Sixth Berkeley Symposium, III, Univ. of California Press, pp. 579-614 (1972)

Mollison, D.: Spatial contact models for ecological and epidemic spread. J. Roy. Statist. Soc. B39, 283-326 (1977)

Mollison, D., Kuulasmaa, K.: Spatial epidemic models: theory and simulations. In: Bacon, P. J. (ed.) Population dynamics of rabies in wildlife, pp. 291-309. New York: Academic Press 1985

Mollison, D.: Modelling biological invasions: chance, explanation, prediction. Philos. Trans. R. Soc. Lond. B, 314, 675-693 (1986)

Mooney, H. A., Drake, J. A. (eds.): Ecology of biological invasions of North America and Hawaii. (Ecological Studies, vol. 58) Berlin Heidelberg New York: Springer 1986

Nobel, J. V.: Geographic and temporal development of plagues. Nature 250, 726-729 (1974)

Okubo, A.: Diffusion-type models for avian range expansion. In: Quellet, H. (ed.) Acta XIX Congress Internationa lis Ornithologici, vol. 1, pp. 1038-1049. National Museum of Natural Sciences, University of Ottawa Press, Ontario, Canada 1988

Othmer, H. G., Dunbar, S. R., Alt, W.: Models of dispersal in biological systems. J. Math. Biol. 26, 263-298 (1988)

Roughgarden, J.: Theory of population genetics and evolutionary ecology: an introduction. New York: MacMillan 1979

Sikes, R. K.: Pathogenesis of rabies in wildlife. I. Comparative effect of varying doses of rabies virus inoculated into foxes and skunks. Am. J. Vet. Res. 23, 1041-1047 (1962)

Skellam, J. G.: Random dispersal in theoretical populations. Biometrica 38, 196-218 (1951)

Smith, A. D. M.: A continuous time dterministic model of temporal rabies. In: Bacon, P. J. (ed.) Population dynamics of rabies in wildlife. New York: Academic Press 1985

Steck, F., Wandeler, A.: The epidemiology of fox rabies in Europe. Epidemiol. Rev. 2, 71-96 (1980)

Thieme, H. R.: A model for the spatial spread of an epidemic. J. Math. Biol 4, 337-351 (1977a)

Thieme, H. R.: The asymptotic behaviour of solutions of nonlinear integral equations. Math. $Z$. 157, 141-154 (1977b)

Thieme, H. R.: Asymptotic estimates of the solutions of non-linear integral equations and asymptotic speeds for the spread of populations. Jal Reine Angew. Math. 306, 94-121 (1979a)

Thieme, H. R.: Density-dependent regulation of spatially distributed populations and their asymptotic speed of spread. J. Math. Biol. 8, 173-187 (1979b)

Van den Bosch, F., Zadoks, J. C., Metz, J. A. J.: Focus expansion in plant disease. I: The constant rate of focus expansion. Phytopathology 78, 54-58 (1988) 
Van den Bosch, F., Zadoks, J. C., Metz, J. A. J.: Focus expansion in plant disease. II: Realistic parameter-sparse models. Phytopathology 78, 59-64 (1988)

Van den Bosch, F., Frinking, H. D., Metz, J. A. J., Zadoks, J. C.: Focus expansion in plant disease. III: Two experimental examples. Phytopathology 78, 919-925 (1988)

Van den Bosch, F., Verhaar, M. A., Buiel, A. A. M., Hoogkamer, W., Zadoks, J. C.: Focus expansion in plant disease. IV: Expansion rates in mixtures of resistant and susceptible hosts. Phytopathology, in press

Van den Bosch, F., Hengeveld, R., Metz, J. A. J.: Analysing animal range expansion. Preprint (1988)

Verkaik, A. J.: The muskrat in the Netherlands. Proceedings of the Koninklijke Nederlandse Akademie van Wetenschappen 90, 67-72 (1987)

Vincent, J. P., Quéré, J. P.: Quelques donées sur la reproduction et sur la dynamique des populations du rat musqué dans le nord de la France. Ann. Zool. Ecol. anim. 4, 395-415 (1972)

Watt, K. E. F.: Ecology and resource management. New York: McGraw-Hill 1968

Weinberger, H. F.: Asymptotic behaviour of a model in population genetics. In: Chadam, J. M. (ed.) Nonlinear partial differential equations and applications. (Lect. Notes in Maths., vol. 648, pp. 47-98) Berlin Heidelber New York: Springer 1978

Weinberger, H. F.: Long-time behaviour of a class of biological models. SIAM J. Math. Anal. 13, 353-396 (1982)

Williamson, E. J.: The distribution of larvae of randomly moving insects. Aust. J. Biol. Sci. 14, 598-604 (1961)

Williamson, M. H., Brown, K. C.: The analysis and modelling of British invasions. Phil. Trans. R. Soc. London B314, 505-522 (1986)

Wolfe, M. S.: The current status and prospects of multiline cultivars and variety mixtures for disease resistance. Annu. Rev. Phytopathol. 23, $251-273$ (1985)

Zadoks, J. C., Kampmeijer, P.: The role of crop populations and their development, illustrated by means of a simulator Epimul 76. Ann. N.Y. Acad. Sci. 287, 164-190 (1977)

Zawolek, M. W.: A physical theory of focus development in plant disease. Agric. Univ. Wageningen Papers. Pudoc, in press

Received November 21, 1988; received in revised form December 8, 1989 